\title{
IFN-I response timing relative to virus replication determines MERS coronavirus infection outcomes
}

\author{
Rudragouda Channappanavar, ${ }^{1,2,3}$ Anthony R. Fehr, ${ }^{1}$ Jian Zheng, ${ }^{1}$ Christine Wohlford-Lenane, ${ }^{4}$ Juan E. Abrahante, ${ }^{5}$ \\ Matthias Mack, ${ }^{6}$ Ramakrishna Sompallae, ${ }^{7}$ Paul B. McCray Jr., ${ }^{1,4}$ David K. Meyerholz, ${ }^{7}$ and Stanley Perlman ${ }^{1,4,8}$ \\ 'Department of Microbiology and Immunology, University of lowa, lowa City, lowa, USA. ${ }^{2}$ Department of Acute and Tertiary Care, and ${ }^{3}$ Department of Microbiology and Immunology, University of Tennessee \\ Health Sciences Center, Memphis, Tennessee, USA. ${ }^{4}$ Department of Pediatrics, University of lowa, lowa City, lowa, USA. ${ }^{5}$ University of Minnesota Informatics Institute, University of Minnesota, Minneapolis, \\ Minnesota, USA. ${ }^{6}$ Department of Internal Medicine, University Hospital Regensburg, Regensburg, Germany. ${ }^{7}$ Department of Pathology, University of lowa, lowa City, lowa, USA. ${ }^{8}$ State Key Laboratory of \\ Respiratory Disease, National Clinical Research Center for Respiratory Disease, Guangzhou Institute of Respiratory Health, the First Affiliated Hospital of Guangzhou Medical University, Guangzhou, China.
}

\begin{abstract}
Type 1 IFNs (IFN-I) generally protect mammalian hosts from virus infections, but in some cases, IFN-I is pathogenic. Because IFN-I is protective, it is commonly used to treat virus infections for which no specific approved drug or vaccine is available. The Middle East respiratory syndrome-coronavirus (MERS-CoV) is such an infection, yet little is known about the role of IFN-I in this setting. Here, we show that IFN-I signaling is protective during MERS-CoV infection. Blocking IFN-I signaling resulted in delayed virus clearance, enhanced neutrophil infiltration, and impaired MERS-CoV-specific T cell responses. Notably, IFN-I administration within 1 day after infection (before virus titers peak) protected mice from lethal infection, despite a decrease in IFN-stimulated gene (ISC) and inflammatory cytokine gene expression. In contrast, delayed IFN- $\beta$ treatment failed to effectively inhibit virus replication; increased infiltration and activation of monocytes, macrophages, and neutrophils in the lungs; and enhanced proinflammatory cytokine expression, resulting in fatal pneumonia in an otherwise sublethal infection. Together, these results suggest that the relative timing of the IFN-I response and maximal virus replication is key in determining outcomes, at least in infected mice. By extension, IFN- $\alpha \beta$ or combination therapy may need to be used cautiously to treat viral infections in clinical settings.
\end{abstract}

\section{Introduction}

Emerging and reemerging highly pathogenic zoonotic viruses are a significant threat to global public health (1-3). This threat is illustrated by the appearance of several highly pathogenic respiratory viruses such as severe acute respiratory syndromecoronavirus (SARS-CoV), Middle East respiratory syndrome-CoV (MERS-CoV), and pathogenic strains of influenza viruses $(1,3)$. MERS-CoV is a novel human $\mathrm{CoV}$ that emerged in 2012 in the Middle East and has since spread to 27 countries, with the majority of cases recorded in the Middle East and South Korea $(4,5)$. Since its identification, MERS-CoV has infected 2374 individuals and caused 823 deaths (as of February 28, 2019) (6). MERS-CoV continues to infect human populations in the Middle East and as a result remains a public health threat. Additionally, isolation of MERS-CoV from camels and identification of SARS- and MERSlike CoVs in bats make it likely that these viruses will continue to emerge and cause additional outbreaks $(7,8)$. Despite the high morbidity and mortality rates associated with MERS, the limited number of autopsy studies has hindered our understanding of MERS in humans. Additionally, MERS-CoV causes mild to moderate disease in nonhuman primates and other nonhuman hosts,

Conflict of interest: The authors have declared that no conflict of interest exists. Copyright: (c) 2019, American Society for Clinical Investigation.

Submitted: November 21, 2018; Accepted: June 4, 2019; Published: July 29, 2019.

Reference information: J Clin Invest. 2019;129(9):3625-3639.

https://doi.org/10.1172/JCI126363. making it challenging to study MERS pathogenesis (9). Among several animal models used to study MERS-CoV pathogenesis, we and others have shown that infection of human dipeptidyl peptidase 4-knockin (hDPP4-KI) mice with a mouse-adapted strain of MERS-CoV (MERS-CoV-MA) replicates several features of MERS in humans $(10,11)$.

While pathogenic human CoVs (hCoVs) are known to cause severe pneumonia, the mechanistic basis of their high morbidity and mortality is incompletely understood. Rapid virus replication reaching high titers and associated enhanced inflammatory responses are believed to contribute to severe pneumonia (12-14). The innate antiviral response, particularly production of IFN-I (IFN- $\alpha$ and IFN- $\beta$ ), constitutes the first line of defense against multiple virus infections. IFN-I mediates antiviral effects by directly inhibiting virus replication and indirectly modulating the host immune response to virus infection, both of which are mediated by induction of IFN-stimulated genes (ISGs) (15, 16). Because of its antiviral effects, IFN-I has been used in trials in combination with other antiviral agents to prevent and treat emerging and reemerging virus infections for which no approved drugs are available (17-20). However, results from these trials have yielded inconsistent results. In addition, other studies indicated that IFN-I has pathogenic effects during acute and chronic infections $(14,21-24)$. Together, these findings suggest that the relationship between virus replication and or related pathogenesis and the kinetics of IFN expression, whether endogenous or after exogenous administration, contributed to the variability 

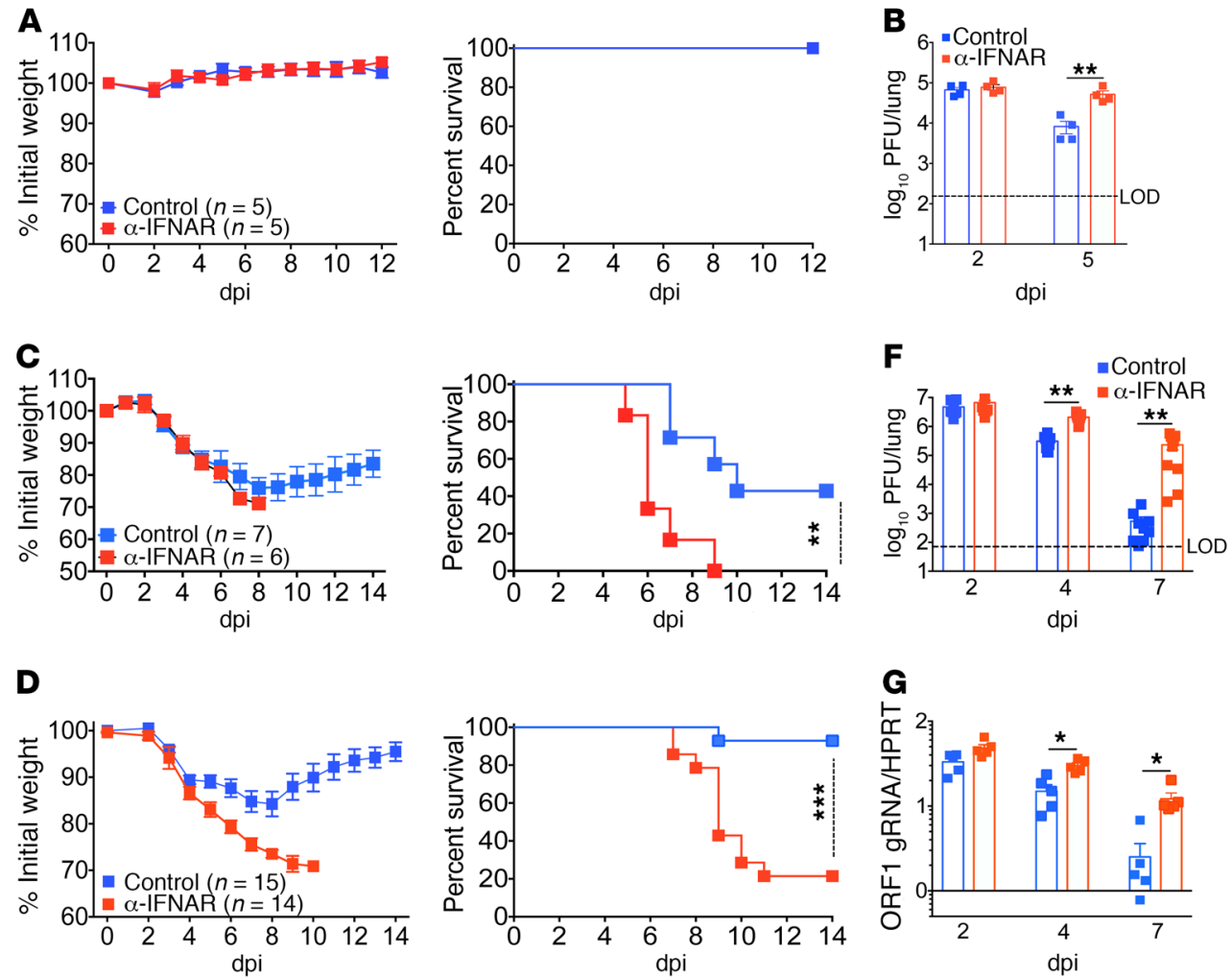

\section{E}
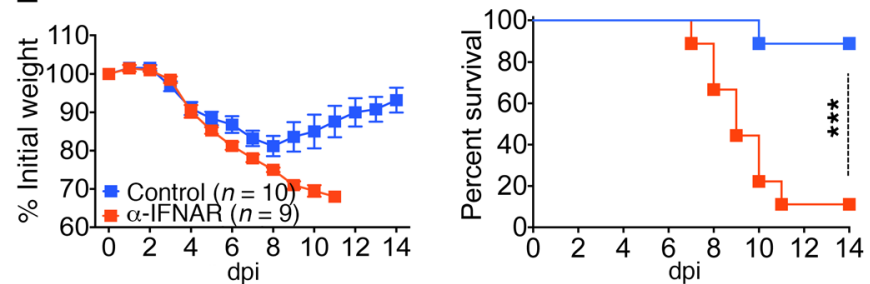

H

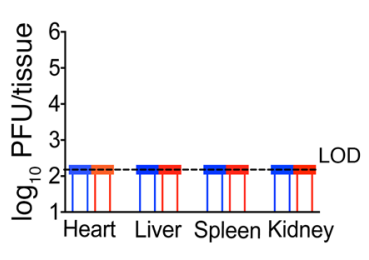

I
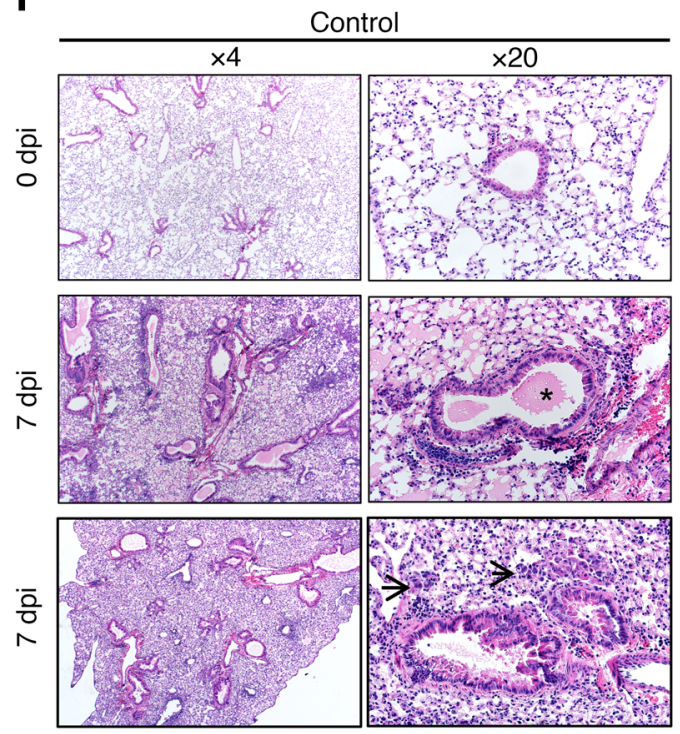

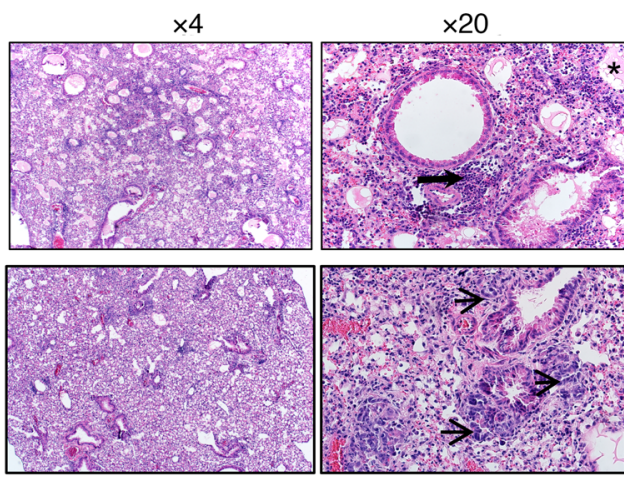

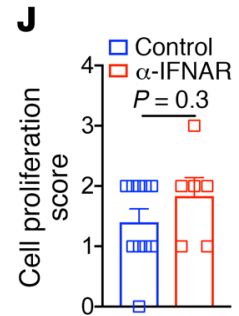

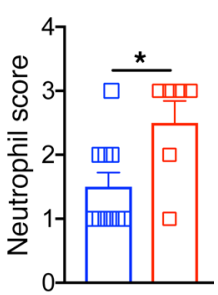


Figure 1. IFN-I signaling is protective during MERS-CoV infection. (A) Percentage of initial weight and survival of control- and $\alpha$-IFNAR-treated mice after i.n. infection with $1 \times 10^{5}$ PFU MERS-CoV-EMC. (B) MERS-CoVEMC titers in the lungs determined by plaque assay at 2 and $5 \mathrm{dpi}$. (C-E) Percentage of initial weight and survival of control and $\alpha$-IFNAR-treated mice after i.n. infection with 500 PFU (C) or 200-250 PFU (D, female mice; E, male mice) MERS-CoV-MA. (F and $\mathbf{G})$ MERS-CoV titers as determined by plaque assays (F) and gRNA levels (G) in the lungs of control and $\alpha$-IFNARtreated mice infected with 200 to 250 PFU MERS-CoV-MA. (H) MERSCoV-MA titers in the indicated organs at $4 \mathrm{dpi}$ as determined by plaque assay. (I) Representative $\mathrm{H} \& \mathrm{E}$ staining of lungs collected from naive (top panel) and MERS-CoV-MA-challenged mice at $7 \mathrm{dpi}$, demonstrating lung edema and neutrophil infiltration (middle panels) and cellular proliferation (bottom panels). Original magnification, $\times 4$ and $\times 20$. Arrows point to neutrophils; arrowheads show cell proliferation; asterisk indicates edema. (J) Summary scores for cellular proliferation and neutrophil distribution. Data are representative of $\mathbf{2}$ independent experiments (A-C, and $\mathbf{G}-\mathbf{I}$ ) or were pooled from 2 independent experiments ( $\mathbf{D}-\mathbf{F}$ and $\mathbf{J})(n=3$ to 5 mice/ group). ${ }^{*} P \leq 0.05,{ }^{* *} P \leq 0.01$, and ${ }^{* * *} P \leq 0.001$ (B, $\mathbf{F}-\mathbf{H}$, and $\mathbf{J}$ ), by 2 -tailed Student's $t$ test. Statistical significance for survival studies (A and $\mathbf{C}-\mathbf{E}$ ) was calculated using a log-rank (Mantel-Cox) test with a $95 \% \mathrm{Cl}$ and a $P$ value of less than 0.05 considered significant.

of outcomes. IFN-I therapy has been used to treat patients with severe respiratory disease caused by CoVs, with similarly inconsistent outcomes (25). In particular, IFN-I treatment of patients with MERS failed to improve survival $(17,26,27)$. For example, in one study, IFN treatment prolonged survival when assessed at 14 days, but not at 28 days (17).

Here, we used a mouse model of MERS-CoV infection to investigate the mechanistic basis for the protective and pathogenic effects of early and delayed IFN responses, respectively, during viral pneumonia. We show that, unlike in SARS-CoVinfected mice, IFN-I signaling is protective during MERS-CoV infection and is specifically required for virus clearance. Early exogenous administration of recombinant IFN- $\beta$ (rIFN- $\beta$ ) completely protected mice from lethal MERS-CoV infection by inhibiting virus replication and inflammatory cytokine production. Conversely, delayed rIFN- $\beta$ therapy caused a striking increase in IFN, ISG, and inflammatory cytokine levels, resulting in fatal disease in an otherwise sublethal infection. These results demonstrate that the timing of IFN- $\alpha \beta$ receptor (IFNAR) signaling relative to peak virus replication is a critical determinant of protective and pathogenic immunity and may help explain the disparate results obtained in clinical trials.

\section{Results}

IFN-I signaling is essential for host protection during MERS-CoV infection. To investigate whether the absence of clinical disease in hDPP4-KI mice infected with $1 \times 10^{5} \mathrm{PFU}$ of a human isolate of MERS-CoV (Erasmus Medical Center/2012 [EMC/2012]) strain) was dependent on IFN-I signaling, we treated infected hDPP4KI mice with control or anti-IFNAR ( $\alpha$-IFNAR) antibody. Neither $\alpha$-IFNAR-treated nor control mice lost weight, and there were no differences in survival (Figure 1A). However, $\alpha$-IFNAR-treated mice had a significantly higher viral load 5 days postinfection (dpi) compared with control mice (Figure 1B). We next infected control and $\alpha$-IFNAR-treated mice with an $\mathrm{LD}_{50}$ dose of MERS-CoV-MA (500 PFU). Although we observed a $60 \%$ mortality rate in the control mice, $100 \%$ of the $\alpha$-IFNAR-treated mice succumbed to MERS-CoV-MA infection (Figure 1C). Further, following infection with a sublethal (200-250 PFU) dose of MERS-CoV-MA, the $\alpha$-IFNAR-treated mice lost more weight than did the control mice (>25\%) and had reduced survival rates (Figure 1, D and E). Lung virus titers and viral genomic RNA (gRNA) levels were similar in both groups of mice at $2 \mathrm{dpi}$ (Figure 1, F and G). However, at both $4 \mathrm{dpi}$ and $7 \mathrm{dpi}$, we observed higher virus titers in the lungs of $\alpha$-IFNAR-treated mice compared with titers in control mice (Figure $1, F$ and $G$ ). To assess whether delayed virus clearance resulted in an extrapulmonary spread, we measured virus titers in other organs. We did not detect MERS-CoV-MA in heart, liver, spleen, or kidney tissue (Figure 1H), suggesting that even in the absence of IFN-I signaling, MERS-CoV infection is limited to the lungs in hDPP4-KI mice. We also performed lung histology studies and observed enhanced neutrophil infiltration and cellular hyperplasia in $\alpha$-IFNAR-treated mice compared with control mice (Figure 1 , I and J). However, because the kinetics of disease progression overlapped between the control and $\alpha$-IFNAR-treated mice, only the difference in the numbers of neutrophils reached statistical significance. Together, these results suggest that IFN-I signaling is required for virus clearance and host protection during MERS$\mathrm{CoV}$ infection.

TLR-7 is the primary sensor of MERS-CoV RNA in airway epithelial cells. A delayed IFN-I response relative to peak lung virus titers correlated with severe disease in a mouse model of SARS (14). Consequently, we next compared the kinetics of the IFN response and MERS-CoV-MA replication in hDPP4-KI mice. Unlike in SARS-CoV-infected mice (14), we observed simultaneous peaks of MERS-CoV-MA replication and IFN (IFN- $\alpha \beta$ and IFN- $\lambda$ ) responses in the lungs of hDPP4-Ki mice (Figure 2A), implying that both early and simultaneous IFN responses protect the host from virus infection. Next, we examined innate sensors essential for MERS-CoV RNA detection and IFN induction. Previous studies using mouse CoVs showed that TLR7/MyD88 and MDA5/MAVS pathways are critical mediators of CoV RNA sensing and IFN induction in plasmacytoid DCs (pDCs) and bone marrow-derived macrophages (BMDMs), respectively $(28,29)$. Since pattern recognition receptor (PRR) expression varies with the cell type $(30,31)$ and hCoVs mainly infect airway epithelial cells $(13,32-35)$, we examined whether one or both of these pathways are essential for IFN induction in epithelial cells. To do this, we transduced WT C57BL/6 (B6) and $\mathrm{MAVS}^{-/-} \mathrm{B} 6$, and WT $\mathrm{BALB} / \mathrm{c}$ and $T L R 7^{-/-} \mathrm{BALB} / \mathrm{c}$ mice with adenovirus 5-expressing human DPP4 (Ad5-hDPP4), as previously described (36). Ad5hDPP 4 transduces the majority of airway and alveolar epithelial cells, and most of these Ad5-hDPP4-transduced cells are subsequently infected with MERS-CoV-EMC (36). Additionally, Ad5hDPP4-transduced B6 and BALB/c mice show similar disease following MERS-CoV-EMC infection (36). Since hDPP4-KI mice with deletion of MAVS or TLR7 are not available, and since only transduced cells are infected with MERS-CoV (36), we used Ad5hDPP4-transduced B6, BALB/c, $\mathrm{MAVS}^{-/-}$, and $T L R 7^{-/-}$mice to study sensors required for IFN induction. In addition, intranasally (i.n.) administered Ad5 efficiently transduces epithelial cells (37), allowing us to investigate viral RNA sensing specifically in epithelial cells (36). Six days after transduction, we infected 
A
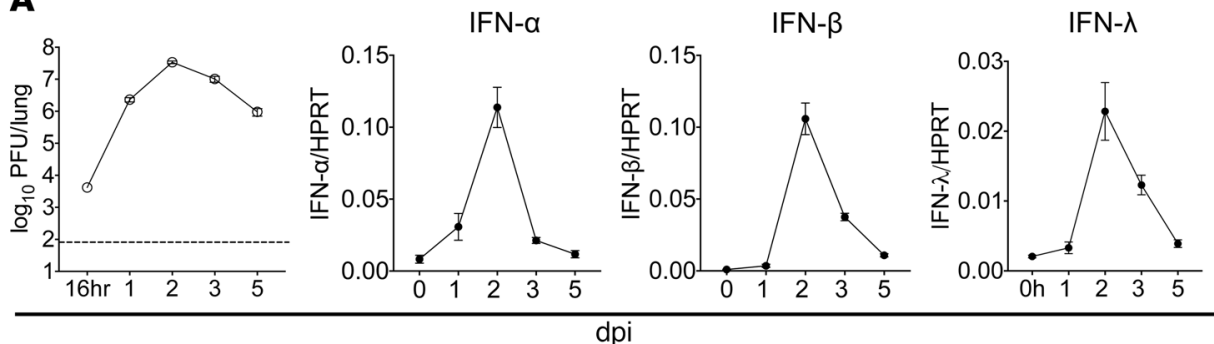

$\mathrm{dp}$
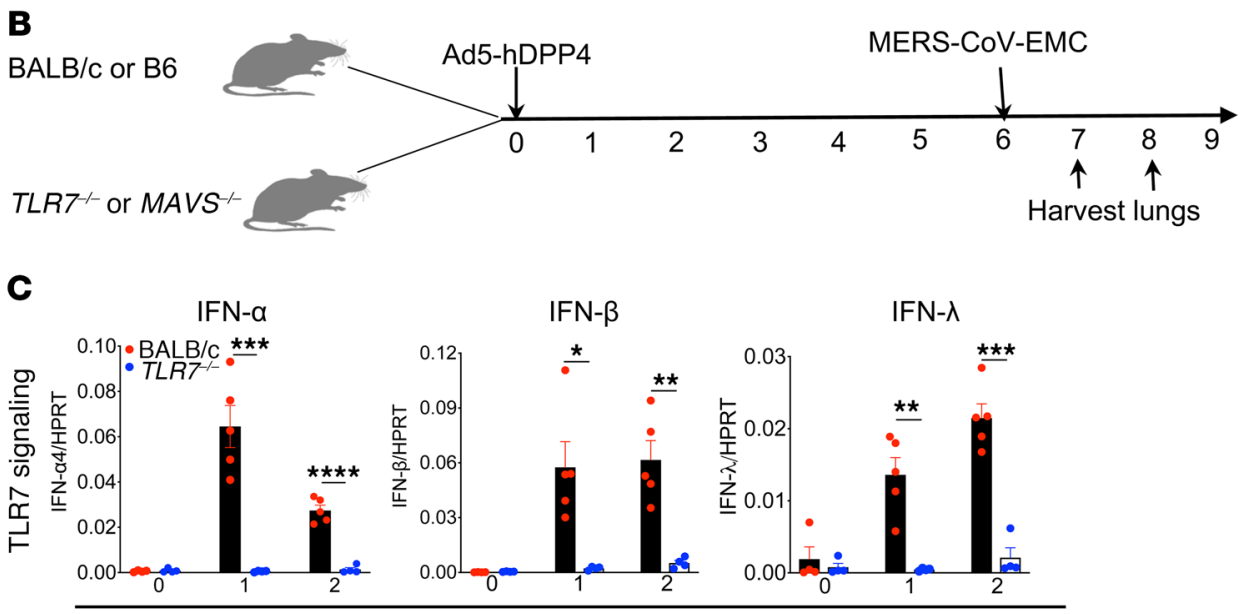

dpi
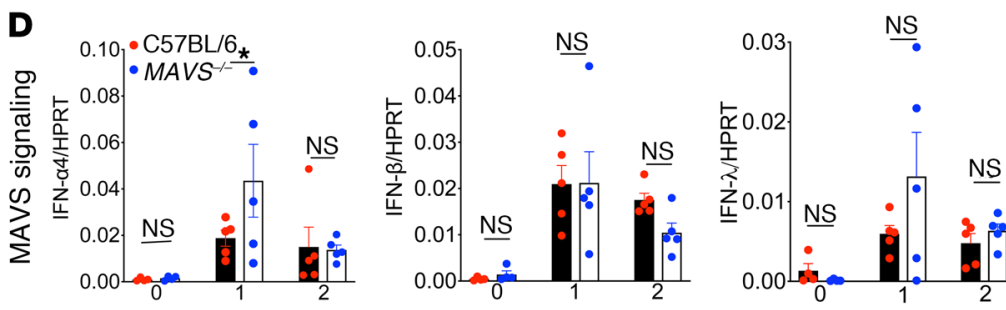

dpi
Figure 2. Viral RNA sensing and IFNproduction in MERS-CoV-infected mice. (A) Lung virus titers and IFN mRNA levels in lungs from hDPP4-KI mice at different time points after MERS-CoV-MA infection (200-250 PFU). (B) Schematic diagram demonstrating the experimental plan to examine MERS-CoV-MA RNA sensing and IFN induction. (C and $\mathbf{D}$ )

Transcript levels of IFN- $\alpha 4$, IFN- $\beta$, and IFN- $\lambda$ relative to the housekeeping gene HPRT in the lungs at 0,1 , and 2 dpi with MERS-CoV-EMC infection ( $\left.1 \times 10^{5} \mathrm{PFU}\right)$ in Ad5-hDPP4-transduced WT and TLR7-/mice (C) and Ad5-hDPP4-transduced WT and MAVS $^{-/-}$mice (D). Data are representative of 2 independent experiments, with 4 to 5 mice/group/experiment. ${ }^{*} P \leq 0.05,{ }^{*} P \leq 0.01$, and ${ }^{* * *} P \leq 0.001$, ${ }^{* * * *} P \leq 0.0001$ by 2 -tailed Student's $t$ test.
WT and KO mice with MERS-CoV-EMC virus and measured lung IFN transcript levels at 1 and $2 \mathrm{dpi}$ (Figure 2B). We found significantly reduced IFN- $\alpha$, IFN $-\beta$, and IFN- $\lambda$ expression in the lungs of $T L R 7^{-/}$mice compared with expression levels in BALB/c mice (Figure 2C). In contrast, IFN levels were similar or even elevated in $\mathrm{MAVS}^{-/-}$mice compared with levels in B6 mice (Figure 2D). Like MERS-CoV infection, SARS-CoV predominantly infects lung epithelial cells and abortively infects hematopoietic cells $(13,38,39)$. We therefore infected $T L R 7^{-1-}$ and $\mathrm{MAVS}^{-/-}$mice and their respective WT controls with a mouse-adapted strain of SARS-CoV (MA15) as another approach to determine the relevant virus-sensing molecule. Infection with MA15 showed that signaling of both TLR7 and MAVS was essential for IFN induction in the lungs, as demonstrated by reduced IFN- $\alpha$, IFN- $\beta$, and IFN- $\lambda$ mRNA levels in the lungs from $T L R 7^{-/-}$ and $\mathrm{MAVS}^{-/-}$mice compared with lungs from WT controls (Supplemental Figure 1, A and B). These results indicate that TLR7/ MyD88 signaling is an important mediator of the IFN response in lung epithelial cells during MERS-CoV infection but that MAVS signaling, based on the results following SARS-CoV infection, is important after infection of nonepithelial cells.
Blocking IFN-I signaling modulates inflammatory monocytes and macrophages and neutrophil responses to MERS-CoV infection. Severe disease in $\mathrm{hCoV}$ infection is generally characterized by an excessive innate immune response. In particular, a robust infiltration of inflammatory monocytes and macrophages (IMMs) $\left(\mathrm{CD} 45^{+} \mathrm{CD} 11 \mathrm{~b}^{+} \mathrm{Ly}_{6} \mathrm{C}^{\text {hi }}\right)$ occurs in severe SARS, contributing to mortality $(34,40,41)$. Since IMM migration is IFN dependent, we next measured immune cell infiltration and proinflammatory cytokine and chemokine mRNA levels in the lungs of control and $\alpha$-IFNAR-treated hDPP4-KI mice. As expected, there was a significant reduction in the total number of IMMs in the lungs of $\alpha$-IFNAR-treated mice at 2 and 4 dpi compared with controls (Figure $3, \mathrm{~A}$ and $\mathrm{B})$. In contrast, although there was no difference in the total number of neutrophils $\left(\mathrm{CD} 45^{+} \mathrm{CD} 11 \mathrm{~b}^{+} \mathrm{Ly} 6 \mathrm{G}^{\mathrm{hi}}\right)$ in the lungs 2 and 4 dpi between groups, $\alpha$-IFNAR-treated mice had higher numbers of neutrophils by 7 dpi compared with control mice (Figure 3, C and D). Further, neutrophils in the lungs of $\alpha$-IFNARtreated mice produced significantly higher levels of the proinflammatory cytokines TNF, IL-1 $\beta$, and IL-6, as well as increased levels of iNOS compared with control mice (Supplemental Figure 2B). To examine whether IFN-I signaling alters other immune cells in 
A

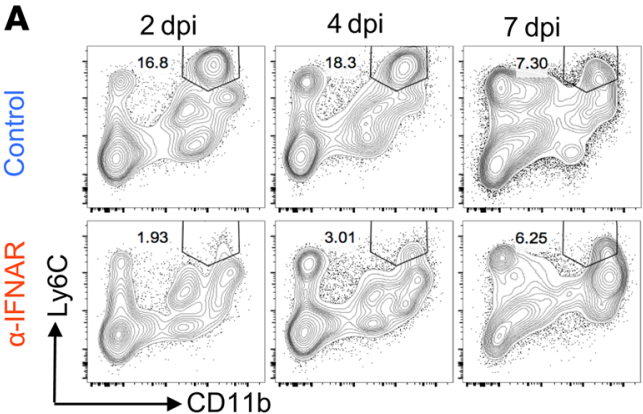

C

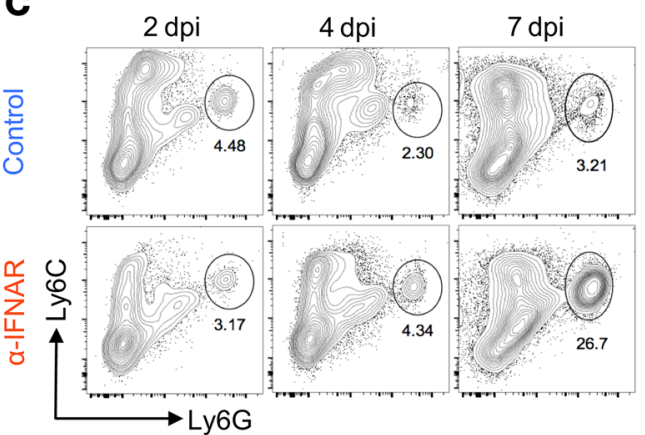

B

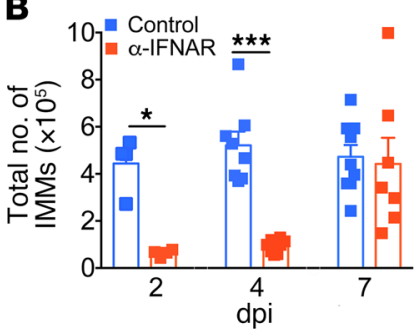

D

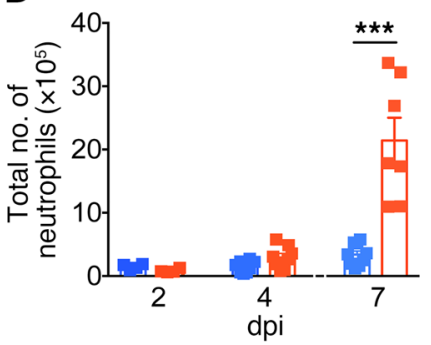

Figure 3. Immune cell and cytokine and chemokine responses to MERS-CoV-MA infection. (A) Representative FACS plots and (B) quantification of CD11b+Ly6Chi IMMs in the lungs of control- and $\alpha$-IFNAR-treated mice following MERS-CoV-MA infection (200-250 PFU). (C) Representative FACS plots and (D) quantification of Ly6C ${ }^{\text {hi }}$ Ly6G+ neutrophils in the lungs of control and $\alpha$-IFNAR-treated mice following MERS-CoV-MA infection. (E and F) mRNA levels of IFNs, ISGs, and cytokines and chemokines on different days after MERSCoV-MA infection in control and $\alpha$-IFNAR lungs. (G) MERS-CoV-specific CD4 ${ }^{+}$and $\mathrm{CD}^{+} \mathrm{T}$ cells in lungs from control and $\alpha$-IFNAR-treated mice were identified on the basis of IFN- $\gamma$ production in response to stimulation with either N99 or 51165 peptide 7 dpi. (H) MERSCoV-MA titers in control and $\mathrm{CD}^{+}$and $\mathrm{CD}^{+}$ T cell-depleted lungs $7 \mathrm{dpi}$. Data were either pooled from 2 independent experiments (B and $\mathbf{D}$ ) or are representative of 2 independent experiments (E-H) with 3 to 5 mice/group/ experiment. ${ }^{*} P \leq 0.05$, ${ }^{*} P \leq 0.01$, and ${ }^{*}{ }^{*} P$ $\leq 0.001$, by 2 -tailed Student's $t$ test.
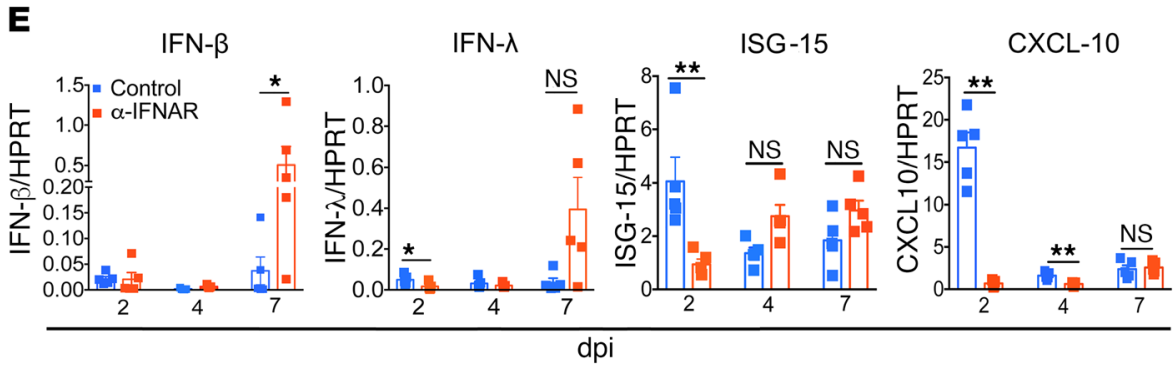

$\mathbf{F}$

CCL-2
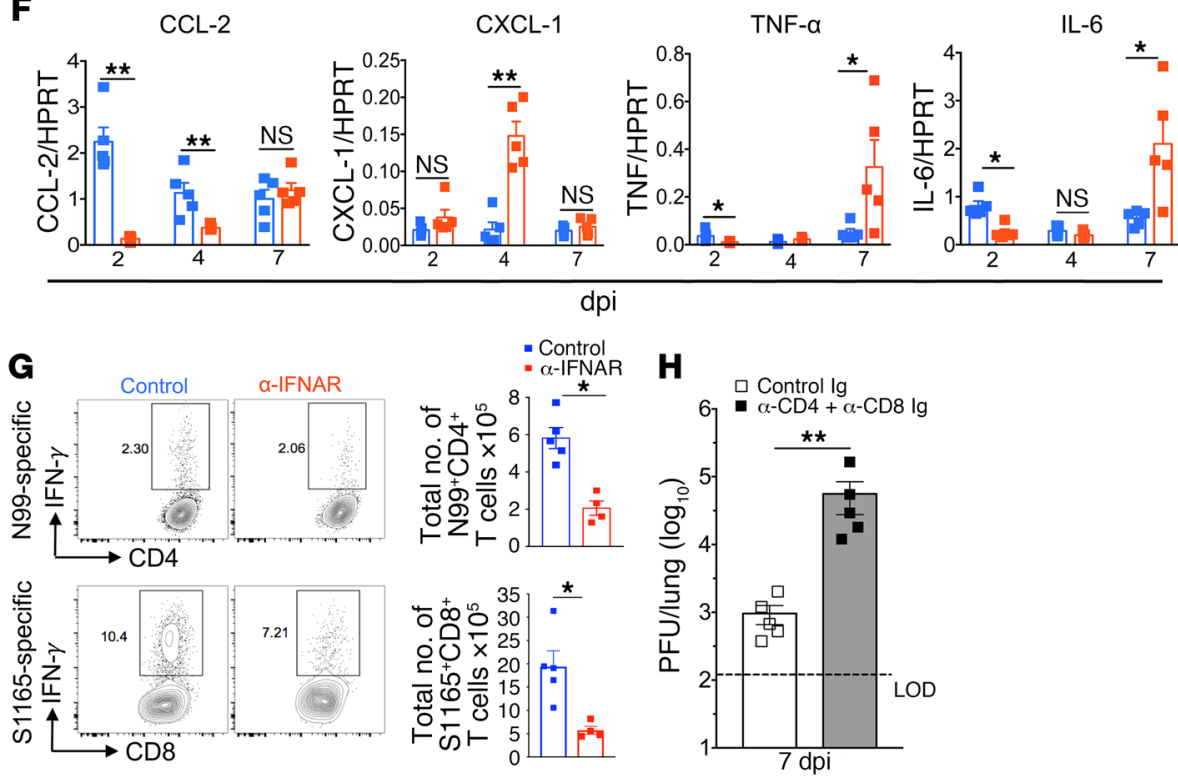

the lungs, we measured total numbers of alveolar macrophages (AMs), conventional DCs, and NK cells. Although AM numbers were similar in control and $\alpha$-IFNAR mice early after infection, we found that they were increased in $\alpha$-IFNAR-treated mice by day 7 dpi (Supplemental Figure 2A). Total DC and NK cell numbers in the lungs were lower in $\alpha$-IFNAR-treated mice at 2 and 4 dpi, respectively (Supplemental Figure 2A), suggesting an effect of IFN-I signaling on the accumulation of these cells. 
A
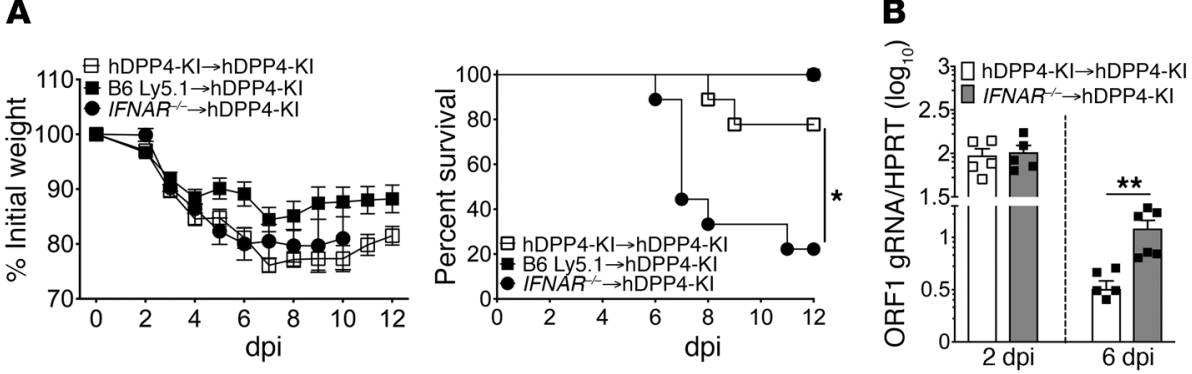

C
IFN- $\alpha$

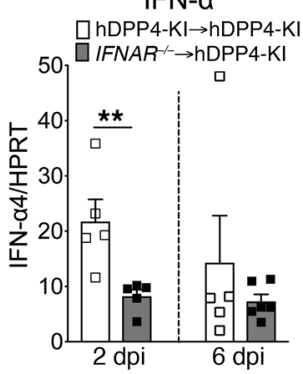

IFN- $\beta$

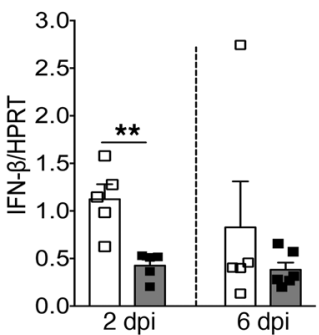

IFN- $\lambda$

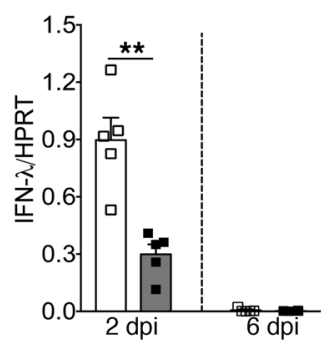

ISG-15

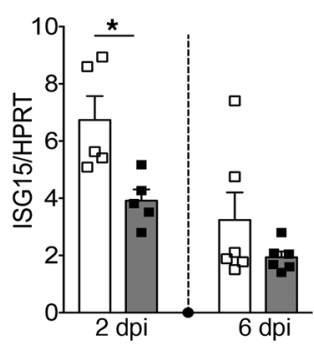

D

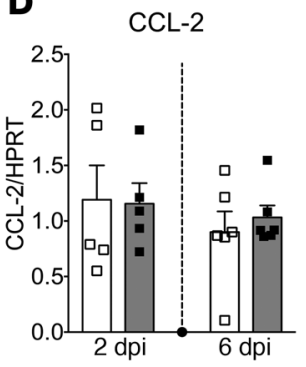

CXCL-1

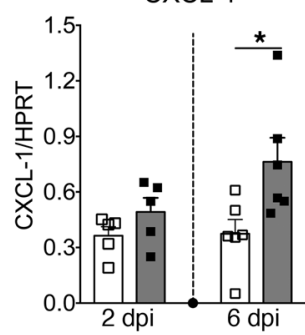

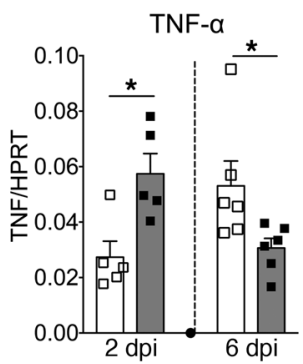

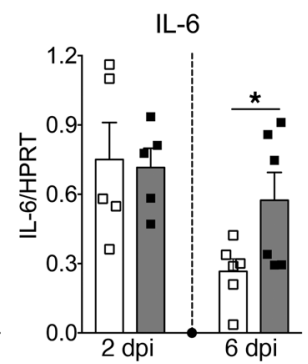

Figure 4. IFNAR signaling on hematopoietic cells is essential for host protection from MERS-CoV-MA infection. (A) Percentage of initial weight and survival of BM chimeric mice after MERS-CoV-MA challenge (500 PFU, i.n.). Data were pooled from 2 independent experiments with 3 to 5 mice/group/experiment. (B) MERS-CoV gRNA levels in lungs from $B M$ chimeric mice at 2 and $6 \mathrm{dpi}$. (C and D) mRNA levels of IFNs and ISGs (C) and inflammatory cytokines and chemokines (D) in lungs from MERS-CoV-infected BM chimeric mice (hDPP4-KI hDPP4-KI and IFNAR ${ }^{-/-}$hDPP4-KI). (A) Weight loss and survival curves show pooled data from 2 independent experiments $(n=$ 4 to 5 mice/group/experiment). Statistical significance for survival studies in $\mathbf{A}$ was calculated using the log-rank (Mantel-Cox) test with a $95 \% \mathrm{Cl}$ and a $P$ value of less than 0.05 considered significant. (B-D) Graphs show pooled data from 2 independent experiments ( $n=2-3$ mice/group/experiment). ${ }^{*} P \leq 0.05$ and ${ }^{* *} P \leq 0.01$, by 2 -tailed Student's $t$ test.
Furthermore, lungs from $\alpha$-IFNAR-treated mice had significantly lower transcript levels of IFN- $\lambda$, ISGs (ISG-15 and CXCL10), and inflammatory cytokines and chemokines (TNF, IL-6, CCL-2) at early time points (2 dpi, and in some cases, $4 \mathrm{dpi}$ ) compared with controls (Figure 3, E and F). Levels of CXCL-1, a neutrophil chemoattractant, were significantly increased on 4 dpi in $\alpha$-IFNAR-treated mice, thereby preceding the increased neutrophil accumulation detected by histology and flow cytometric analysis at $7 \mathrm{dpi}$. (Figure $1 \mathrm{I}$ and Figure 3, C and D). In contrast, mRNA levels of IFN- $\beta$ and IFN- $\lambda$ and the proinflammatory cytokines TNF and IL- 6 were significantly higher by 7 dpi in $\alpha$-IFNAR-treated mice compared with levels in control mice (Figure 3, E and F), possibly due to a higher virus burden (Figure 1, F and G) and the accumulation of highly activated neutrophils (Supplemental Figure 2B). Further, blocking IFN-I signaling led to reduced numbers of MERS-CoV-specific CD $4^{+}$ and $\mathrm{CD}^{+} \mathrm{T}$ cells in the lungs (Figure $3 \mathrm{G}$ ). To ascertain their role in MERS-CoV infection, we depleted $\mathrm{CD}^{+}$and $\mathrm{CD}^{+} \mathrm{T}$ cells using a combined $\alpha$-CD 4 and $\alpha-C D 8$ antibody. As shown in Figure $3 \mathrm{H}$, depletion of $\mathrm{T}$ cells impaired MERS-CoV clearance from the lungs in comparison with control antibody treatment, suggesting that the reduction in virus-specific $\mathrm{T}$ cells contributed to delayed virus clearance in $\alpha$-IFNAR-treated mice (Figure $1, F$ and $G$ ). Collectively, these results indicate that blocking IFN-I signaling promotes inflammatory neutrophil accumu- lation, alters cytokine and chemokine responses, and impairs virus-specific $\mathrm{T}$ cell responses.

IFN-I signaling in hematopoietic cells is essential for virus clearance and host protection. IFN-I signaling in epithelial cells, hematopoietic cells, or both could be required for protection from a viral infection $(42,43)$. Notably, infection of BM-derived cells from hDPP4-KI mice with MERS-CoV failed to produce infectious virus (Supplemental Figure 3). To examine the role of hematopoietic IFNAR signaling in MERS, we created BM chimeric mice as described previously (14). Briefly, BM from B6-Ly5.1, hDPP4-KI, or IFNAR ${ }^{-/}$donor mice was adoptively transferred into hDPP4-KI, B6-Ly5.1, or IFNAR ${ }^{-/-}$recipient mice, and chimeras were infected with a sublethal dose of MERS-CoV-MA. As expected, B6-Ly5.1 $\rightarrow$ B6-Ly5.1 $\rightarrow$ IFNAR ${ }^{--} \rightarrow$ B6-Ly5.1 mice did not develop MERS, because they lacked hDPP4 expression. Although MERS-CoVMA-challenged hDPP4-KI $\rightarrow$ hDPP4-KI mice lost more weight than did B6-Ly5.1 $\rightarrow$ hDPP4-KI mice, their survival was similar following infection (Figure 4A). However, the lack of IFN-I signaling in hematopoietic cells in IFNAR ${ }^{-/-} \rightarrow$ hDPP4-KI mice significantly decreased survival compared with hDPP4-KI mice that received hDPP4-KI or B6-Ly5.1 BM (Figure 4A). Further, although MERS-CoV gRNA levels were similar in hDPP4-KI hDPP4-KI and $\mathrm{IFNAR}^{-/-} \rightarrow \mathrm{hDPP} 4-\mathrm{KI}$ mice at $2 \mathrm{dpi}$, we observed a 4 - to 5 -fold increase in MERS-CoV RNA levels in IFNAR $R^{--} \rightarrow$ hDPP4-KI lungs compared with levels in hDPP4-KI $\rightarrow$ hDPP4-KI lungs at 6 
A

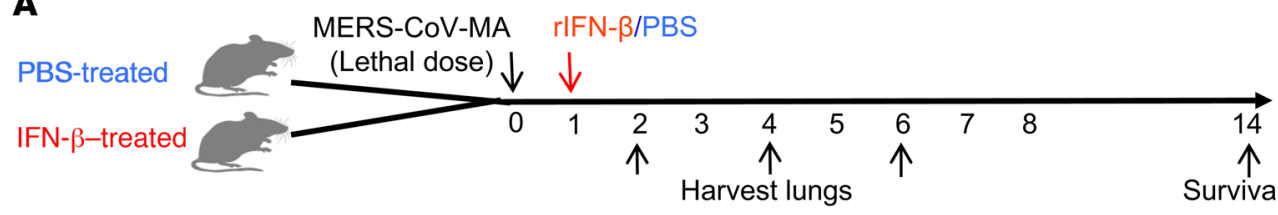

C

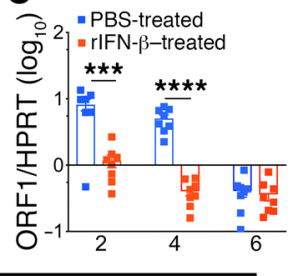

B

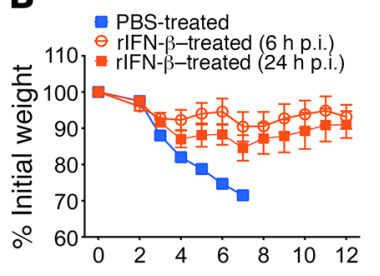

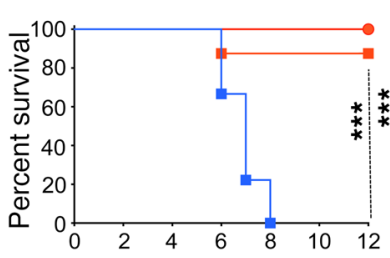

dpi
D
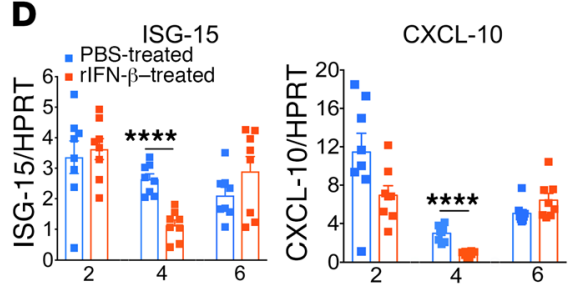

E
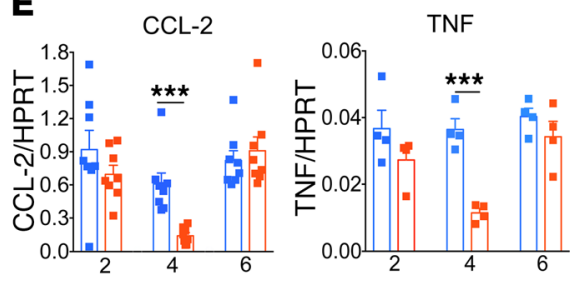

dpi
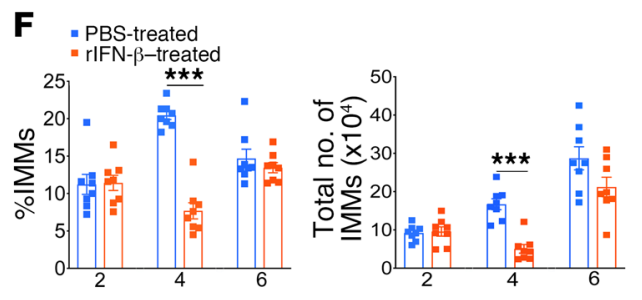

G

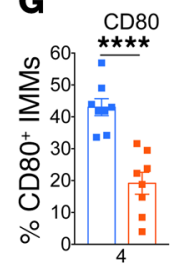

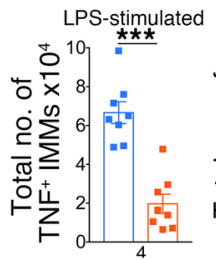

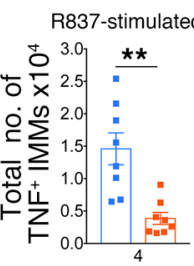

dpi
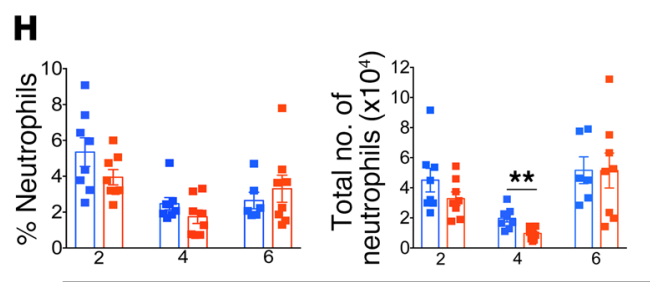

I
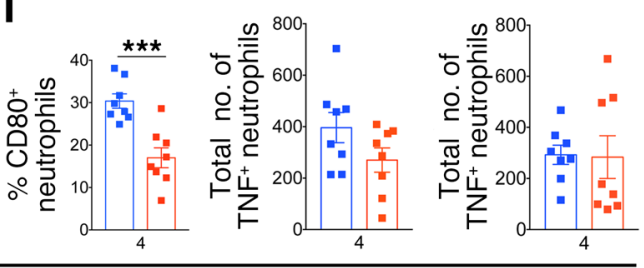

dpi

dpi (Figure 4B). We also measured mRNA levels of IFN, ISG, and other inflammatory cytokines in the chimeric mice. We found that loss of IFN-I signaling in hematopoietic cells caused reduced transcript levels of IFN- $\alpha$ and IFN- $\beta$, IFN- $\lambda$, and ISG-15 in the lungs at 2 dpi and increased proinflammatory cytokine and chemokine transcript levels at 2 dpi (TNF) and 6 dpi (IL-6 and CXCL-1) (Figure $4, \mathrm{C}$ and D). These data suggest that IFNAR signaling in hematopoietic cells is essential for virus clearance and protection against mortality following MERS-CoV infection.

Early treatment with rIFN- $\beta$ provides complete protection from lethal MERS-CoV infection. Results shown in this study, in conjunction with those of previous studies (14), indicate that the timing of the peak IFN response determines disease outcome. To further investigate the relationship of IFN timing to disease outcome
Figure 5. Early treatment with rIFN- $\beta$ protects the host by inhibiting MERS-CoV-MA replication and reducing inflammation. (A) Schematic diagram of the experimental plan to examine the effects of early rIFN- $\beta$ treatment following MERS-CoV-MA infection. (B) Percentage of initial weight and survival of MERS-CoV-MA-infected hDPP4-KI mice that received PBS or rIFN- $\beta$ treatment 6 hours p.i. or 1 dpi. (c) MERS-CoV gRNA levels in the lungs of mice treated with PBS or rIFN- $\beta$ ( 1 dpi, early treatment) at 2, 4 , and 6 dpi. ( $\mathbf{D}$ and $\mathbf{E}$ ) mRNA levels of ISGs (D) and inflammatory cytokines and chemokines (E) in PBS- or rIFN- $\beta$-treated ( 1 dpi) mice. ( $F$ and H) Percentage and total number of IMMs and neutrophils in the lungs of PBS- or rIFN- $\beta$-treated mice determined at 4 dpi. (G and I) Percentage of CD80-expressing and total number of $\mathrm{TNF}^{+} \mathrm{IMMs}$ and neutrophils in lungs from PBS- and rIFN- $\beta$-treated mice (1 dpi) at $4 \mathrm{dpi}$. Data were pooled from 2 separate experiments (B-I) or are representative of 2 separate experiments (E, right panel) ( $n=4-5$ mice/group/experiment). ${ }^{* *} P \leq 0.01$, ${ }^{* *} P \leq 0.001$, and ${ }^{* * *} P$ $\leq 0.0001$, by 2 -tailed Student's $t$ test. Statistical significance for survival studies in B was calculated using a log-rank (Mantel-Cox) test with a $95 \% \mathrm{Cl}$ and $\mathrm{P}$ value of less than 0.05 considered significant. during respiratory virus infection, we treated lethally infected (750 PFU MERS-CoV-MA) hDPP4-KI mice with 750 U rIFN- $\beta$ or PBS at either 6 hours p.i. (hpi) or 1 dpi (Figure 5A). These time points are before peak virus replication occurs, and the rIFN- $\beta$ dose is approximately equivalent to that used in humans (44, 45). As expected, all PBS-treated mice succumbed to infection, whereas mice treated with rIFN- $\beta$ at either 6 hpi or 1 dpi were protected from lethal MERS (Figure $5 \mathrm{~B}$ ). rIFN- $\beta$ treatment significantly reduced MERS-CoV-MA gRNA expression in the lungs at 2 and 4 dpi (Figure $5 C$ ). Surprisingly, the expression of ISGs was downregulated by 4 dpi in the rIFN- $\beta$-treated group compared with expression in the PBS control group (Figure 5D). Likewise, we detected reduced levels of TNF and CCL-2 at 4 dpi in the rIFN- $\beta-$ treated group compared with levels in the control mice (Figure 
A
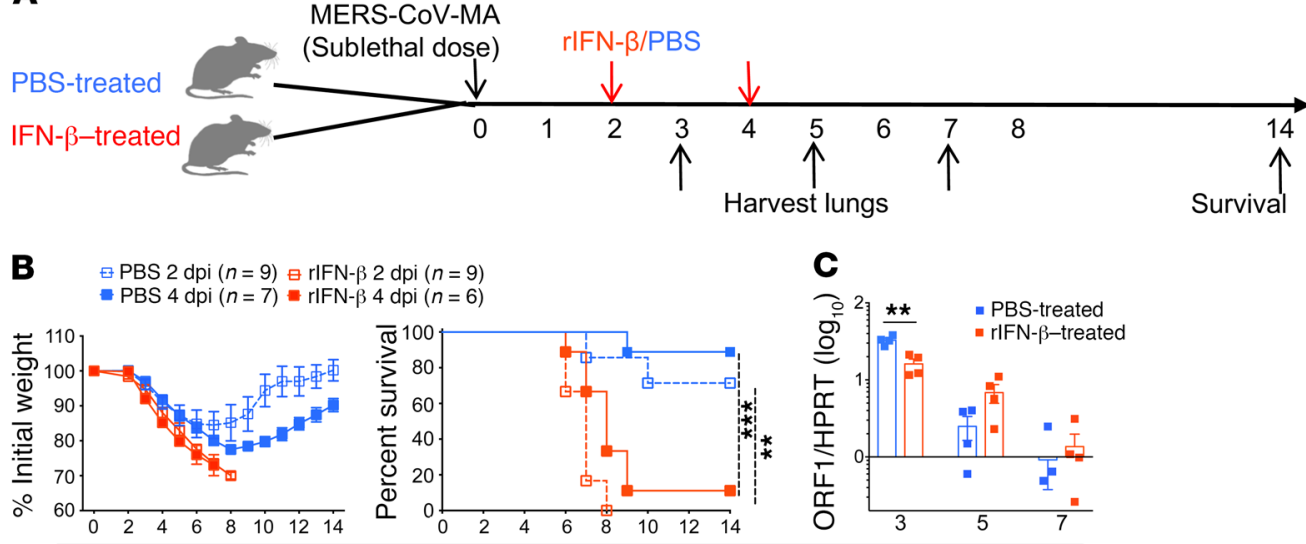

C
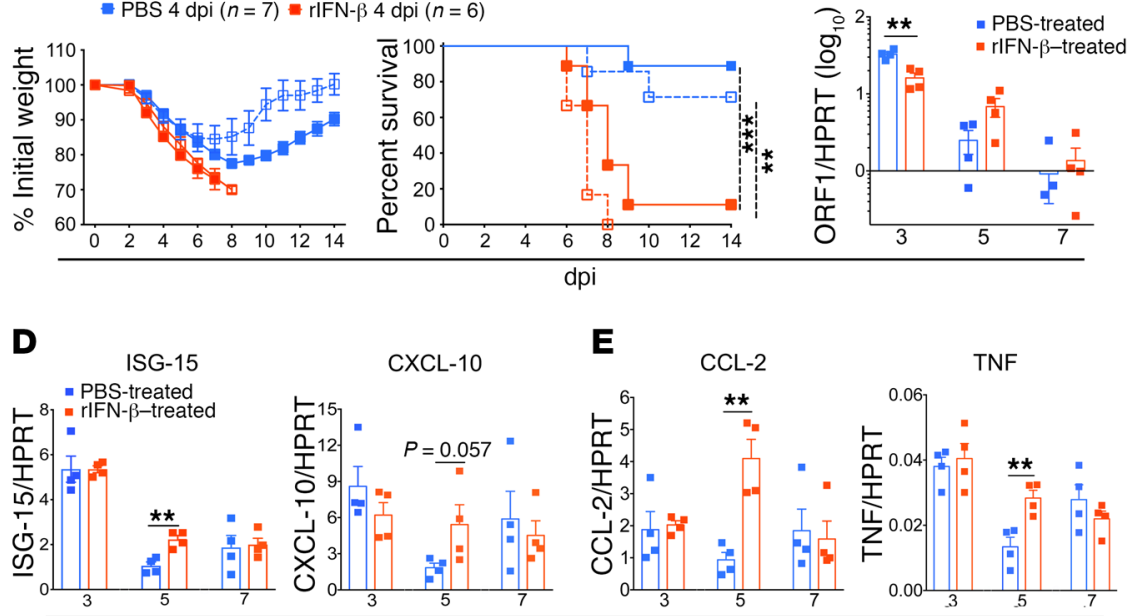

dpi
$\mathbf{F}$

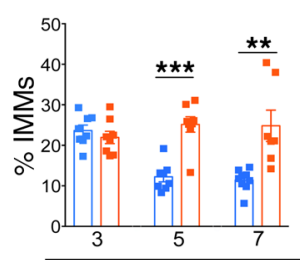

G

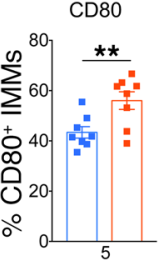

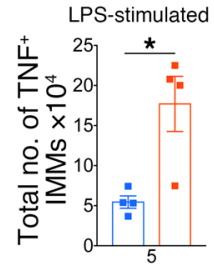

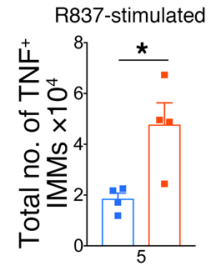

dpi
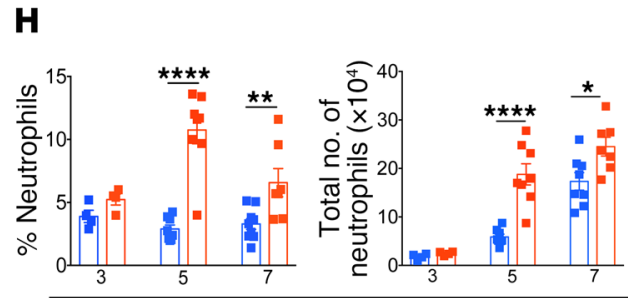

I
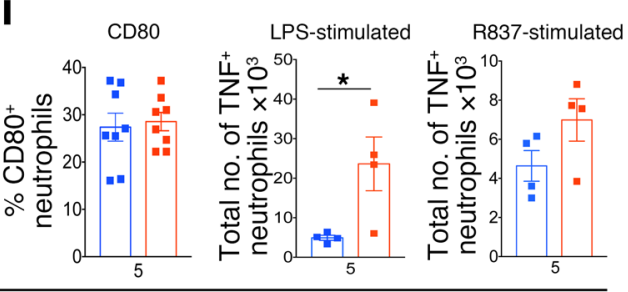

Figure 6. Delayed IFN treatment promotes inflammation and mortality in MERS-CoV-MA-

infected mice. (A) Schematic of the experimental plan to examine the effect of delayed rIFN- $\beta$ treatment. (B) Percentage of initial weight and survival of MERS-CoV-MA-infected hDPP4-KI mice treated with PBS or rIFN- $\beta$ at 2 or 4 dpi. (C) MERS-CoV titers in the lungs at 3, 5, and $7 \mathrm{dpi}$ in mice treated with PBS or rIFN- $\beta$ (2 dpi, delayed treatment). ( $\mathbf{D}$ and E) mRNA levels of ISCs and inflammatory cytokines and chemokines in the lungs of PBS- or rIFN- $\beta$ treated (2 dpi, delayed treatment) mice. (F and $\mathbf{H}$ ) Frequency and number of IMMs and neutrophils in the lungs of PBS- or rIFN- $\beta$-treated mice (2 dpi). (G and I) Percentage of CD80-expressing and total number of $\mathrm{TNF}^{+}$IMMs and neutrophils at 5 dpi in PBS- and rIFN- $\beta$-treated mice (2 dpi). (J) Percentage of initial weight and survival of MERS-CoVMA-infected (200 PFU) hDPP4-KI mice treated with rIFN- $\beta$ (2 dpi) and either $\alpha$-CCR2 antibody or a control antibody (2 dpi and $4 \mathrm{dpi}$ ). Data were pooled from 2 separate experiments ( $\mathbf{B}$ and $\mathbf{F}-\mathbf{H}$, left panels, I, left panel, and J) or are representative of 2 separate experiments (E, G, and I, right 2 panels) ( $n=3-5$ mice/group/experiment). Data were analyzed using a 2-tailed Student's $t$ test with ${ }^{*} P \leq 0.05,{ }^{*} P$ $\leq 0.01,{ }^{* *} P \leq 0.001$, and ${ }^{* * * *} P \leq$ 0.0001 . Statistical significance for survival studies (B, right) was calculated using the log-rank (MantelCox) test, with a $95 \% \mathrm{Cl}$ and a $P$ value of less than 0.05 considered significant.

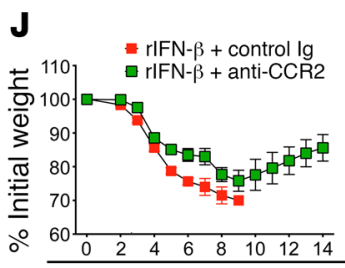

dpi

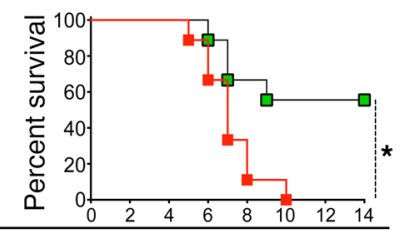

dpi
5E). The levels of ISG-15, CXCL-10, CCL-2, and TNF were not different between the groups at either 2 or 6 dpi. Early rIFN- $\beta$ treatment also reduced the frequency and number of lung-infiltrating IMMs and the number of neutrophils at $4 \mathrm{dpi}$ but not at 2 or $6 \mathrm{dpi}$ (Figure 5, F and H). Moreover, IMMs and neutrophils in the lungs of rIFN- $\beta$-treated mice at 4 dpi were less activated, as shown by reduced expression of CD80 and lower numbers of $\mathrm{TNF}^{+} \mathrm{IMMs}$ after stimulation with TLR4 (LPS) or TLR7 (R837) ligands (Figure 5, G and I). Collectively, these results demonstrate that early treatment with rIFN- $\beta$ protects the host from mortality by inhib- 
A
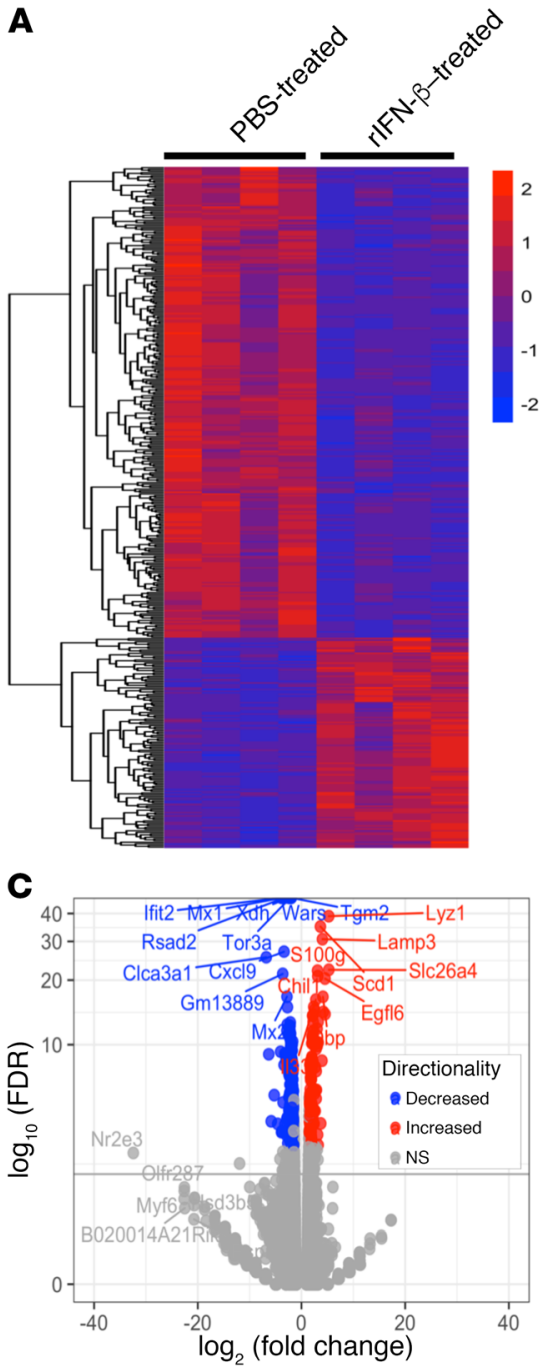

E

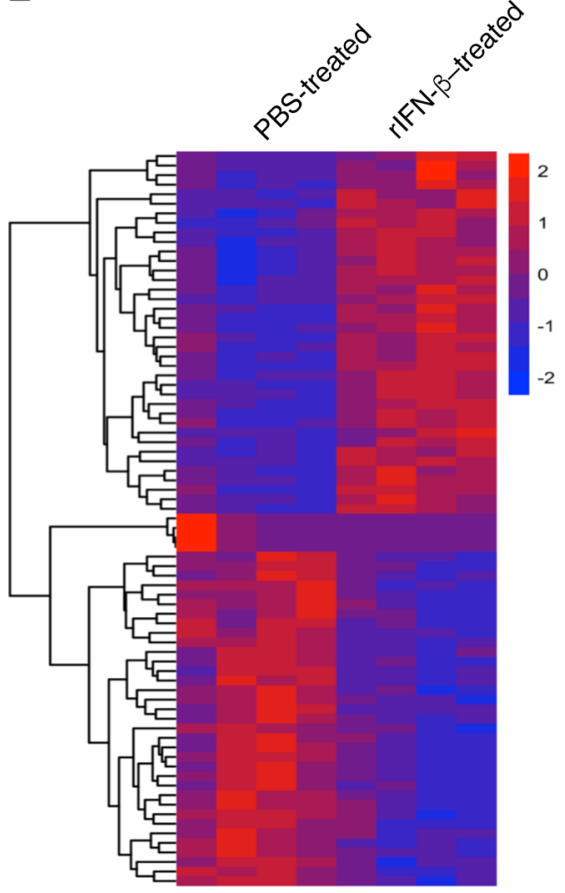

B
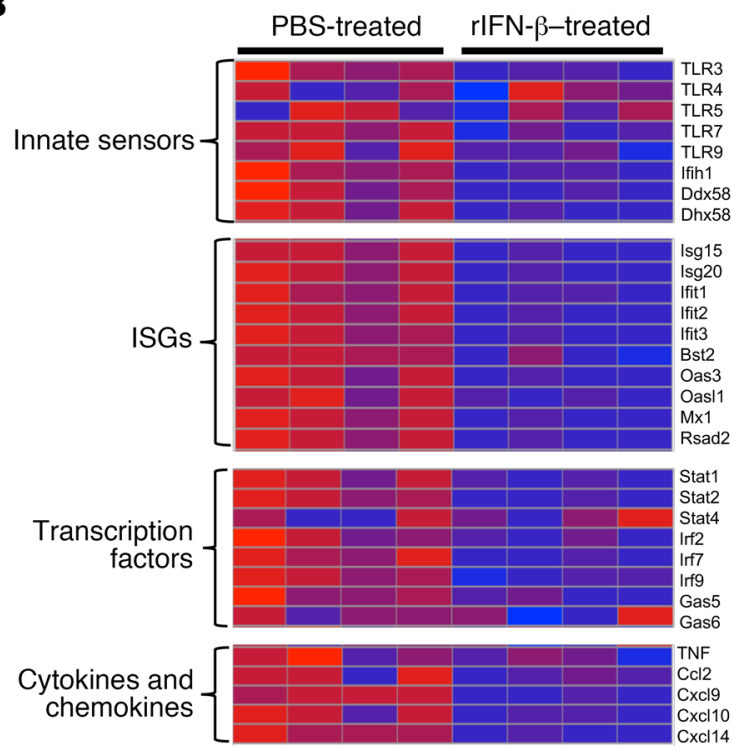

D

Decreased expression of genes involved in immuno-inflammatory pathways in rIFN- $\beta$-treated group

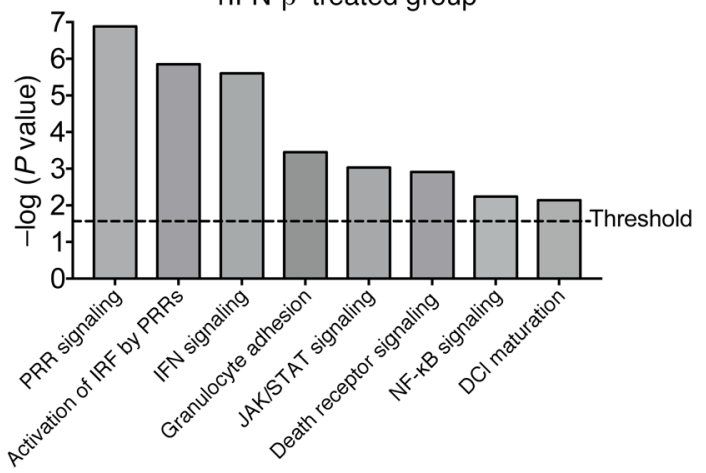

$\mathbf{F}$

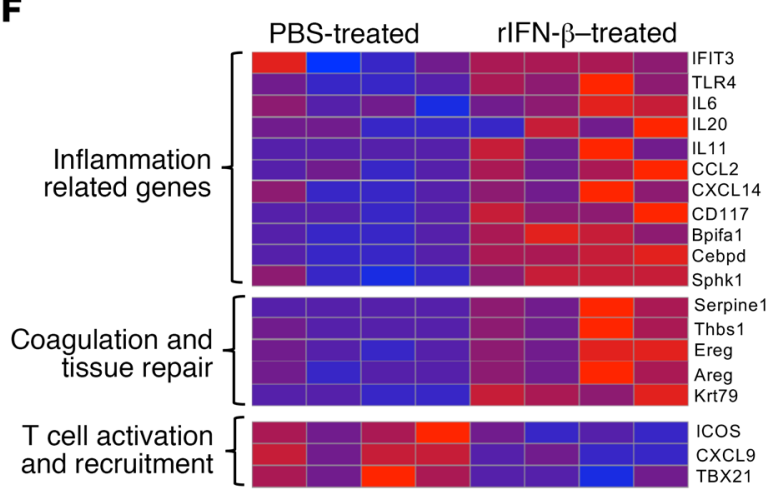

G

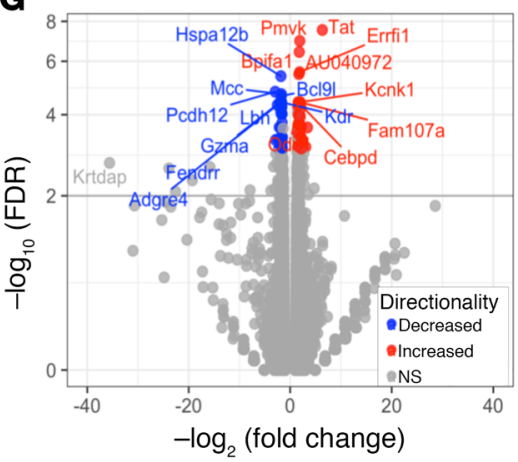

Figure 7. RNA-Seq analyses of gene expression profile in MERS-CoV-MA-infected lungs with early or delayed rIFN- $\beta$ treatment. hDPP4-KI mice infected with MERS-CoV-MA (200 PFU) were treated with 750 U rIFN- $\beta$ at 1 dpi (early, A-D) or 2 dpi (delayed, E-H). Mice were euthanized 2 days after rIFN- $\beta$ treatment ( $3 \mathrm{dpi}$ for mice in the early rIFN- $\beta$ treatment and $4 \mathrm{dpi}$ for mice in the delayed rIFN- $\beta$ treatment groups). RNA isolated from total lungs was used for RNA-Seq studies. ( $A$ and $\mathbf{B}$ ) Heatmaps of differential gene expression profiles in lungs from PBS-treated compared with early rIFN- $\beta$-treated mice display all transcripts (A) and selected innate immune pathways (B). (C) Volcano plot with $\log _{2}$ fold change and $\log _{10} P$ values for differentially expressed genes in lungs from mice treated with PBS compared with mice treated early with rIFN- $\beta$. (D) Major innate immune pathways differentially regulated in control and early rIFN- $\beta$ treatment groups identified by pathway analysis. Results show decreased expression of several proinflammatory mediators after rIFN- $\beta$ treatment. (E and $\mathbf{F})$ Heatmaps of differential gene expression in lungs from mice treated with PBS compared with mice that received delayed rIFN- $\beta$ treatment, displaying all transcripts (E) and selected innate immune genes (F). (G) Volcano plot with $\log _{2}$ fold change and $\log _{10} P$ values for differentially expressed genes in lungs from PBS-treated compared with delayed rIFN- $\beta$ treated mice. Red indicates upregulation; blue indicates downregulation. Data were derived from 4 mice per group, with a FDR-adjusted $P$ value of less than 0.001 and $a \log _{2}$ fold change of greater than 1.5 . 
iting virus replication and suppressing deleterious inflammatory responses to MERS-CoV infection.

Delayed IFN therapy is detrimental to the host during MERS$\mathrm{CoV}$ infection. We next evaluated the effect of delayed rIFN- $\beta$ therapy (relative to peak virus replication) on MERS pathogenesis. We infected hDPP4-KI mice with a sublethal dose of MERSCoV-MA and subsequently treated them with PBS or rIFN- $\beta$ at 2 or 4 dpi (Figure 6A). Mice treated with rIFN- $\beta$ at 2 and 4 dpi showed increased weight loss and reduced survival compared with PBS-treated mice, even when the treatment began as early as 2 dpi (Figure 6B), confirming that delayed IFNAR signaling is in fact detrimental. Further, delayed rIFN- $\beta$ treatment caused lethal disease in mice sublethally infected with SARS-CoV (Supplemental Figure 4A), indicating that the lethal effects of delayed IFN-I signaling are not pathogen specific. In contrast to the effects of early rIFN- $\beta$ administration (Figure $5 \mathrm{C}$ ), mice treated with rIFN- $\beta$ at 2 dpi showed only a 3- to 4 -fold reduction in viral RNA levels at $3 \mathrm{dpi}$, but the levels were similar or trended higher at 5 and 7 dpi compared with levels in PBS-treated mice (Figure 6C). Additionally, rIFN- $\beta$ administration at 2 dpi resulted in significantly higher levels of ISG and inflammatory cytokine mRNA (ISG-15, CXCL-10, CCL-2, and TNF) at 5 dpi compared with levels in PBS controls (Figure 6, D and E). Furthermore, we detected increased frequencies and total numbers of IMMs and neutrophils in the lungs of rIFN- $\beta$-treated animals at 5 and 7 dpi (Figure 6, F and $\mathrm{H})$. Additionally, IMMs and neutrophils in the lungs of rIFN- $\beta$ treated mice were highly activated, as shown by increased CD80 expression and/or the numbers of $\mathrm{TNF}^{+}$IMMs and neutrophils after TLR4 or TLR7 stimulation (Figure 6, G and I). We also observed reduced virus-specific $\mathrm{CD} 4^{+}$and $\mathrm{CD} 8^{+} \mathrm{T}$ cell numbers in the lungs of mice treated with $\operatorname{rIFN}-\beta$ at 2 dpi compared with controls and rIFN- $\beta$ treatment at $1 \mathrm{dpi}$ (Supplemental Figure 4B). To examine whether IMMs cause lung pathology in mice treated with rIFN- $\beta$ at 2 dpi, we depleted IMMs using $\alpha$-CCR 2 antibody at 2 and $4 \mathrm{dpi}$. Mice with IMM depletion were protected from mortality compared with mice that received a control antibody (Figure 6J), demonstrating a causal role for IMMs in lethality following delayed rIFN- $\beta$ therapy. These results, together with our previous study (14), suggest that delayed IFN-I signaling or late treatment with $\mathrm{rIFN}-\beta$ fails to effectively control virus replication and is detrimental to the host during MERS-CoV infection. Furthermore, these findings suggest that IFN-mediated recruitment of IMMs to the lungs contributes to lethality.

Differential gene expression profiles in the lungs following early and late IFN treatment. To identify additional effects of early versus delayed IFN therapy during MERS-CoV infection, we performed RNA-Seq-based transcriptional profiling to study changes in global gene expression in the lungs of infected mice treated with early ( $1 \mathrm{dpi}$ ) or delayed ( $2 \mathrm{dpi}$ ) rIFN- $\beta$. A total of 415 genes were differentially expressed (FDR-adjusted $P<0.001$; $\log _{2}$ fold change $>1.5$ ) in the lungs of mice that received early rIFN- $\beta$ treatment compared with PBS controls (Figure 7A). Notably, the expression levels of genes involved in viral nucleic acid sensing, ISGs, IFNrelated transcription factors, and several proinflammatory cytokines and chemokines were markedly lower in the group that received early rIFN- $\beta$ treatment (Figure 7, B and C). Consistent with this observation, Ingenuity Pathway Analysis (IPA) revealed that PRRs, IFN regulatory factor (IRF) activation by cytosolic receptors, and IFN signaling were all decreased in the group that received early rIFN- $\beta$ treatment (Figure 7D). Fewer genes were upregulated in the lungs treated early with $\operatorname{rIFN}-\beta$, but these included genes related to SOCS1, prostaglandin E2 receptor, sirtuin1, and Irgm1 signaling pathways.

We observed a different set of changes in gene expression profiles when control mice and mice that received late rIFN- $\beta$ treatment were compared. A total of 77 genes were differentially expressed (FDR-adjusted $P<0.001 ; \log _{2}$ fold change $>1.5$ ) in the lungs of mice that received delayed rIFN- $\beta$ treatment compared with PBS control lungs (Figure 7, E and G), and these genes were different than those identified in early rIFN- $\beta$ treatment, with an overlap of only 11 genes. We observed increased expression of TLR4 and inflammatory cytokines (IL-6) and chemokines (CXCL-14) in mice that received delayed rIFN- $\beta$ treatment (Figure $7 F)$. Moreover, IPA revealed an upregulation in the expression of genes involved in complement activation and coagulation (Thbs1 and Serpine1), macrophage activation, and inflammatory pathways in mice with delayed rIFN- $\beta$ treatment (Figure $7 \mathrm{~F}$ and Supplemental Table 1). Collectively, these results demonstrate that early and delayed rIFN- $\beta$ treatment induces distinct gene expression profiles in mice infected with MERS-CoV. Although early rIFN- $\beta$ treatment blunted antiviral and inflammatory gene expression, delayed rIFN- $\beta$ treatment increased the expression of a subset of genes associated with inflammation.

\section{Discussion}

Using a mouse model of MERS, we show that the IFN-I response is crucial for host protection and virus clearance. IFN-I is critical for optimal kinetics of virus clearance and has no effect on maximal titers reached in the lungs (Figure 1, B, F, and G). IFN-I expression and peak MERS-CoV replication occurred simultaneously, resulting in protective $\mathrm{T}$ cell responses in infected lungs. Consistent with this, early IFN-I administration was protective. Conversely, late administration of exogenous IFN-I promoted lethality via recruitment of activated IMMs and neutrophils, augmentation of the proinflammatory cytokine response, and inhibition of an optimal virus-specific $\mathrm{T}$ cell response. Similar results were observed in MERS patients, for whom severe disease correlated with increased numbers of neutrophils and monocytes and higher levels of proinflammatory cytokines, including IFN-I $(46,47)$. These results contrast with those obtained in SARSCoV-infected mice, in which the endogenous IFN-I response was deleterious, such that survival was improved in its absence (14), and in which peak IFN-I expression lagged behind peak virus replication. Together, these data suggest that the timing of the IFN-I response relative to the kinetics of virus replication is critical in determining disease outcomes.

Delayed virus clearance in $\alpha$-IFNAR-treated mice after MERS-CoV infection was probably a consequence of impaired MERS-CoV-specific $\mathrm{CD}^{+}$and $\mathrm{CD}^{+} \mathrm{T}$ cell responses, which were diminished in the absence of IFN-I signaling (Supplemental Figure 2). These results are consistent with those of other studies demonstrating that an optimal IFN-I response is required for the emergence of a protective $\mathrm{T}$ cell response $(48,49)$. Like previous studies of SARS-CoV-infected mice and of other virus pathogens 
$(50,51)$, our results confirm an indispensable role for T cells in virus clearance during MERS-CoV infection. A previous study showed that neither $\mathrm{CD}^{+}$nor $\mathrm{CD}^{+} \mathrm{T}$ cells individually were required for virus clearance in MERS-CoV-infected mice (52); however, our results show that depletion of both $\mathrm{CD} 4^{+}$and $\mathrm{CD} 8^{+}$ $\mathrm{T}$ cells results in suboptimal virus clearance, suggesting some degree of redundancy. Of note, the reduced numbers of IMMs and increased neutrophil infiltration in $\alpha$-IFNAR mice (Figure 3, A and B) may be due to altered IMM and neutrophil chemokine production in the absence of IFN-I signaling (53). Further, the enhanced proinflammatory activity of neutrophils (Supplemental Figure 2B) probably contributed to the increase in proinflammatory cytokine and chemokine expression in the lungs of $\alpha$-IFNAR mice (Figure 3, E and F). Although other recent studies have demonstrated similar increases in neutrophil infiltration (53), the differential regulation of neutrophil proinflammatory activity by IFN-I signaling is relatively unexplored. Collectively, these results suggest that a higher virus burden associated with increased accumulation of inflammatory neutrophils leads to fatal pneumonia in $\alpha$-IFNAR-treated mice.

MERS-CoV replicates poorly but induces strong proinflammatory responses in activated human BM-derived cells $(54,55)$ and replicates in activated human T cells (56). However, our studies show that MERS-CoV failed to replicate in hDPP4-KI BMderived cells (Supplemental Figure 3). Despite these results, our chimera studies demonstrate a protective role for IFNAR signaling in hematopoietic cells. Reduced transcript levels of IFN and ISG in the lungs of hDPP4 mice that received IFNAR ${ }^{-/-} \mathrm{BM}$ (Figure 4C) suggest that IFN and ISG were induced via a feedback amplification of IFNAR signaling in hematopoietic cells (Figure 4, C and D) and subsequently played an essential role in IFN and ISG induction and host protection upon MERS-CoV infection. Parenthetically, although it is not known which hematopoietic cell type, if any, is the dominant source of IFN during MERS-CoV infection, MERS studies in human cells (57) and studies of SARS-CoV in both human hematopoietic cells and mouse models $(14,42)$ suggest that pDCs, and possibly macrophages, are the sources of IFN in infected mice.

Previous studies have demonstrated a critical role for MDA5 in sensing CoV RNA and thereby initiating the IFN-I response (29). The majority of these studies used macrophages infected with a murine $\mathrm{CoV}$, mouse hepatitis virus, which is macrophage tropic $(58,59)$. Since human CoVs predominantly infect airway and alveolar epithelial cells and PRR expression is cell-type specific, we examined the PRRs necessary for IFN production specifically in airway and alveolar epithelial cells using mice transduced with Ad5-hDPP4 and then infected with the human EMC/2012 strain of MERS-CoV. Ad5 predominantly infects epithelial cells and not myeloid cells (37). Results from these studies showed that TLR7/ MyD88 but not MAVS signaling was required for IFN production after infection of lung epithelial cells (Figure 2, A and B). As noted previously, human pDCs are the predominant hematopoietic cell source of IFN-I after MERS-CoV infection (57), and signaling occurs through TLR7. Whether infected epithelial cells or uninfected (bystander) pDCs are most important for TLR7-dependent IFN-I expression in Ad5-hDPP4-transduced cells will require further investigation. These results are similar to those of previous studies that showed more severe clinical disease and slower kinetics of virus clearance in $\mathrm{FNAR}^{-/-}$and $\mathrm{Myd} 88^{-/-}$mice compared with WT and $M A V S^{-/-}$mice after Ad5-hDPP4 transduction and MERSCoV infection (36). These data, in conjunction with other findings, demonstrate that RNA sensing by PRRs is virus specific, as MAVS alone or both MyD88 and MAVS together were essential for IFN and ISG induction in mice infected with respiratory syncytial virus (RSV) or influenza A virus (IAV), respectively $(60,61)$. Additionally, it is likely that immune-evasive mechanisms, such as MERS$\mathrm{CoV}$ 4a protein-mediated inhibition of PACT (a dsRNA-binding protein that activates RIG and MDA5), impair MDA5-mediated IFN-I responses (62).

Early rIFN- $\beta$ therapy dramatically reduced viral gRNA levels in the lungs without an increase in ISG expression after rIFN- $\beta$ treatment ( 2 and 6 dpi) (Figures 5 and 7). This result was unexpected, because the antiviral effects of IFN-Is are primarily mediated by ISGs, which reduce virus burden through a variety of mechanisms (15). Instead, we detected substantial decreases in ISG and inflammatory cytokine and chemokine expression in the lungs at 4 dpi (day 3 after IFN therapy). These decreases in ISGs and inflammatory cytokine expression were not due to reduced viral pathogen-associated molecular patterns (PAMPs), as the levels of these mediators were similar at 2 and $6 \mathrm{dpi}$, despite a reduction in viral gRNA levels (Figure 5C). Our results suggest that early IFN treatment suppresses inflammatory cytokine and chemokine expression, thereby dampening the accumulation and function of inflammatory cells, notably IMMs and neutrophils, in the lungs. These results also reveal an antiinflammatory immunomodulatory role for IFN early during acute virus infection. In complete contrast to the early IFN treatment results, delayed IFN treatment resulted in a robust proinflammatory response and did not improve virus clearance (Figure 6, D-I). Notably, we detected upregulation of genes involved in coagulation molecules, which were shown to contribute to severe disease in SARS-CoV-infected mice (63). Ineffective control of virus replication is likely due to IFN and ISG antagonism by viral proteins, and/or the result of impaired virus-specific $\mathrm{T}$ cell responses $(14,64)$. Although further studies are required to determine whether delayed IFN-I signaling is pathogenic during other viral lung infections, the increased disease severity observed in IAV-infected mice receiving prolonged $\mathrm{rIFN}-\alpha$ treatment supports the idea that this signaling has deleterious effects (65).

Furthermore, the exaggerated inflammatory responses observed in delayed rIFN- $\beta$-treated mice underscore the importance of early IFN therapy during acute virus infection. As in our studies, prophylactic or early therapeutic administration of IFNs during MERS-CoV infection in rhesus macaques provided significant protection (66). However, studies in humans failed to conclusively establish the beneficial effects of rIFN therapy (17, $26,27,67)$, possibly because of delayed administration relative to peak virus titers. Our results and these clinical studies suggest that IFN-I treatment has protective and pathogenic effects that are dependent on the timing of administration relative to infection. We attempted to demonstrate this directly by treating MERS-CoV-infected human airway cells with human IFN-I at different time points after infection and assaying for levels of proinflammatory cytokines and virus titers. However, our results 
were not consistent, as relative levels of virus and proinflammatory cytokines varied between experiments. This variability in expression of inflammatory mediators most likely occurred because IFN-I administered to an experimentally infected animal or patient functions in the complex inflammatory milieu of the lung and acts on both infected and bystander cells. MERS-CoV infection of human airway cells in isolation does not adequately reflect this complexity.

In summary, our results demonstrate a critical role for IFN-I in facilitating virus clearance and host survival during MERS-CoV. Additionally, our results suggest that a delayed IFN response contributes to severe viral lung infections, and as a result, understanding the kinetics of MERS-CoV replication will be critical for developing rational therapeutic approaches. It will also be important to develop assays that provide information about the virological and inflammatory status of specific patients to enable appropriate use of IFN and other therapies. Since the timing of the IFN-I response relative to virus replication is critical in determining disease outcome, IFN or combination therapy should be used cautiously to treat acute viral infections, since the peak for virus replication is generally unknown in patients.

\section{Methods}

\section{Mice and virus}

Specific pathogen-free human DPP4-KI mice were generated as described earlier (10). Male and female mice, 8-12 weeks of age, were used for these studies. B6, B6-Ly5.1, and BALB/c mice were purchased from Charles River Laboratories. $M_{A V S^{-/}}$mice on a B6 background and $T L R 7^{-1-}$ mice on a BALB/c background were a gift from Michael Gale Jr. (University of Washington, Seattle, Washington, USA) and Westley Reeves (University of Florida, Gainesville, Florida, USA, with permission from Shizuo Akira, Osaka University, Osaka, Japan), respectively. $T L R 7^{-/}$mice were re-derived by crossing them with Charles River BALB/c mice (Charles River Laboratories). Mice were bred and maintained at the University of Iowa animal care facility. The MERS-CoV-MA15 strain was generated as described earlier (10). Mice were anesthetized using xylazine-ketamine and i.n. infected with a 200 to $250 \mathrm{PFU}$ (sublethal), $500 \mathrm{PFU}\left(\mathrm{LD}_{50}\right)$, or $1000 \mathrm{PFU}$ (lethal) dose of MERS-CoV-MA in $50 \mu \mathrm{L}$ DMEM. All work with MERS-CoV was conducted at the University of Iowa Biosafety Level 3 (BSL3) and animal BSL3 laboratories.

\section{Lung virus titers}

To obtain tissues for virus titers, mice were euthanized on different days after challenge, lungs were removed and homogenized in PBS, and titers were determined for Vero E81 cells. Cells were fixed with $10 \%$ formaldehyde and stained with crystal violet $3 \mathrm{dpi}$. Virus titers are represented as PFU/lung.

\section{Lung histology}

Animals were anesthetized and transcardially perfused with $10 \mathrm{~mL}$ PBS followed by $5 \mathrm{~mL}$ zinc formalin. Lungs were removed, fixed in zinc formalin, and paraffin embedded. Sections were stained with $\mathrm{H} \& \mathrm{E}$ and examined by light microscopy in a blinded fashion (68). Control and $\alpha$-IFNAR-treated lungs were scored for edema and cell proliferation (e.g., remodeling and repair changes such as fibroplasia and/or epithelial hyperplasia), with scores of $0,1,2,3$, and 4 representing lung areas with $0 \%$, less than $3 \%, 6 \%-33 \%, 33 \%-66 \%$, and more than $66 \%$ detectable proliferation, respectively. Lungs were also scored for neutrophil infiltration, with scores of $0,1,2,3$, and 4 representing areas with $0 \%$, less than $3 \%, 6 \%-33 \%, 33 \%-66 \%$, and more than $66 \%$ of perivascular polymorphonuclear cell distribution, respectively.

\section{Lung cell preparation for FACS analysis}

Mice were sacrificed at the indicated time points and perfused via the right ventricle with $10 \mathrm{~mL}$ PBS. Lungs obtained were minced and digested in HBSS buffer containing 2\% FCS, $25 \mathrm{mM} \mathrm{HEPES,} 1 \mathrm{mg} / \mathrm{mL}$ collagenase D (Roche), and $0.1 \mathrm{mg} / \mathrm{mL}$ DNase (Roche) for 30 minutes at room temperature. Digested tissues were then pressed through a $70-\mu \mathrm{m}$ cell strainer to remove particulate matter to obtain single-cell suspensions. Cells were enumerated by $0.2 \%$ trypan blue exclusion or by a Scepter 2.0 cell counter (MilliporeSigma).

\section{Intracellular cytokine staining}

For intracellular cytokine staining (ICS), $1 \times 10^{6}$ cells per well were incubated at $37^{\circ} \mathrm{C}$ for 6 hours in the presence of $1 \mu \mathrm{g}$ Golgiplug (BD Biosciences). T cell ICS was performed after stimulating lung cells with a $1-\mu \mathrm{M}$ concentration of specific peptide. MERS-CoV-specific $\mathrm{CD}^{+}$and $\mathrm{CD}^{+} \mathrm{T}$ cell responses were identified using peptides corresponding to a previously defined epitope (S1165) (36) and a recently identified (by our lab) epitope (N99, YFYYTGTGPEAALPF), respectively. Neutrophil ICS was performed by incubating lung cells with no stimulation at $37^{\circ} \mathrm{C}$ for 7 hours. IMM ICS was performed on cells stimulated with LPS (100 ng/mL, ) or R837 (TLR7 agonist, $1 \mu \mathrm{g} / \mathrm{mL}$ ). Cells were washed and blocked with $1 \mu \mathrm{g} \alpha$-CD16/ $\alpha$-CD32 antibody and surface stained with the indicated antibodies on ice. Cells were then fixed and permeabilized with Cytofix/Cytoperm Solution (BD Biosciences) and labeled with an $\alpha$-cytokine antibody. All flow cytometric data were acquired on a BD FACSVerse (BD Biosciences) and analyzed with FlowJo software (Tree Star).

\section{Antibodies and rIFN- $\beta$}

For surface and intracellular staining, cells were incubated with the following fluorochrome-labeled antibodies specific for mouse antigens: PECy7 $\alpha$-CD45 (30-F11); FITC $\alpha$-Ly6G (1A8, BD Biosciences); PE/PerCp-Cy5.5 $\alpha$-Ly6C (AL-21 [BD Biosciences] or HK1.4); V450 $\alpha$-CD11b (M1/70); APC $\alpha$-F4/80 (BM8); FITC/PE $\alpha$-CD11c (HL3); $\alpha$-CD80 (16-10A1); $\alpha-C D 4$ (RM4-5); $\alpha$-CD8 $\alpha$ (53-6.7); APC $\alpha-\mathrm{TNF}-\alpha$ (MP6-XT22); APC $\alpha$-IL-6 (MP5-20F3); APC $\alpha$-IL- $\beta$ (NJTEN3); APC $\alpha$-iNOS (CXNFT, BD Biosciences); and PerCp-Cy5.5 $\alpha$-IA/IE (M5/114.15.2) (unless otherwise stated, all from eBioscience). APC/PE $\alpha$-CCR2 (475301) was purchased from R\&D Systems; PE/APC $\alpha$-BST2 (JF051C2.4.1) was obtained from Miltenyi Biotec; and APC-MCP-1 (clone 2H5) was purchased from BioLegend. rIFN- $\beta$ was obtained from PBL Assay Science (catalog 12405-1). For in vivo studies, $750 \mathrm{U}$ rIFN- $\beta$ in PBS was administered i.n. at the indicated time points

\section{In vivo antibody treatments and monocyte and neutrophil depletion}

Young, 7- to 8-week-old BALB/c mice were treated i.p. with blocking $\alpha$-IFNAR monoclonal antibody (clone MAR1-5A3, Bio X Cell) at -6 hours $(750 \mu \mathrm{g})$ and on day $1(250 \mu \mathrm{g})$. The same concentrations of mouse IgG1 (clone MOPC21) were used as a control antibody. For monocyte depletion, $\alpha$-CCR2 antibody (clone MC21, $25 \mu \mathrm{g} /$ dose 
in $250 \mu \mathrm{L}$ PBS, i.p.) (69) was administered at 2 and 4 dpi. A similar concentration of rat-IgG was used as a control antibody. T cells were depleted by treating mice with a combination of $\alpha$-CD4 (clone GK1.5, $250 \mu \mathrm{g} / \mathrm{mouse}$ ) and $\alpha$-CD8 antibody (clone $2.43250 \mu \mathrm{g} / \mathrm{mouse}$ ) on -2 , 0 , and +2 dpi. Control mice received rat Ig at a similar concentration at the indicated time points.

\section{Mouse BM chimeras}

BM cells were extracted from femurs and tibiae of hDPP4, B6, B6-Ly5.1, and IFNAR ${ }^{-/-}$mice (all 6-7 weeks of age) and filtered through a $70-\mu \mathrm{m}$ nylon filter, and $\mathrm{RBC}$ were lysed using ammonium chloride potassium (ACK) buffer. Isolated BM cells $\left(1 \times 10^{7}\right.$ cells) were adoptively transferred into lethally irradiated (10 Gy) hDPP4, B6-Ly5.1, or IFNAR ${ }^{-/}$ mice. Chimeric mice were maintained on water supplemented with antibiotics for 4 weeks to prevent opportunistic infections. Reconstitution was verified 5 weeks after BM transfer by FACS analysis of PBMCs. Six weeks after BM transfer, mice were infected with MERS-CoV (500 PFU) and monitored for weight loss and survival. We used 500 PFU in these experiments, because $\mathrm{BM}$ chimeric mice were relatively resistant to developing disease when infected with 200 to 250 PFU MERS-CoVMA. Lungs were collected in TRIzol at 2 and 6 dpi to determine viral RNA levels and mRNA levels of cytokines and chemokines.

\section{RNA preparation from lungs and BM cells and chemokine estimation by quantitative PCR}

Total lung RNA extracted from lungs at different time points after infection using TRIzol (Invitrogen, Thermo Fisher Scientific) was treated with RQ1 RNAse Free DNAse (Promega) for 10 to 15 minutes at $37^{\circ} \mathrm{C}$ to remove gDNA contamination. cDNA was synthesized using M-MLV reverse transcriptase (Invitrogen, Thermo Fisher Scientific), and mRNA levels were determined after normalizing each sample to HPRT. Specific primer sets used for quantitative PCR (qPCR) were previously described (70-72). The primer sequences used to examine MERS-CoV ORF1a gRNA levels were as follows: forward, 5 '-CCACTACTCCCATTTCGTCAG-3' and reverse, 5'-CAGTATGTGTAGTGCGCATATAAGCA-3'.

\section{RNA-Seq and gene expression profiling of lung samples}

Total lung RNA extracted from lungs at the indicated time points p.i. using TRIzol was treated with RQ1 RNAse-Free DNAse for 10 to $15 \mathrm{~min}$ utes at $37^{\circ} \mathrm{C}$ to remove genomic DNA contamination. RNA-Seq analyses were performed at the University of Minnesota Genomics Center.

Sample quality assessment. RNA isolates were quantified using a fluorimetric RiboGreen assay. Total RNA integrity was assessed using capillary electrophoresis (e.g., Agilent BioAnalyzer 2100), generating an RNA integrity number (RIN). For samples to pass the quality control step, sample RNA quantification needed to be higher than $500 \mathrm{ng}$ and have a RIN of 8 or greater, before they are converted to Illumina sequencing libraries.

Library creation. Total RNA samples were converted to Illumina sequencing libraries using Illumina's TruSeq Stranded mRNA Sample Preparation Kit (catalog RS-122-2103. See https://www.illumina. $\mathrm{com} /$ for a detailed list of kit contents and methods). In summary, 500 ng total RNA was oligo-dT purified using oligo-dT-coated magnetic beads, fragmented, and then reverse transcribed into cDNA. The cDNA was adenylated, ligated to dual-indexed (barcoded) adaptors, and amplified using 15 cycles of PCR. The final library size distri- bution was validated using capillary electrophoresis and quantified using fluorimetry (PicoGreen). Indexed libraries were then normalized and pooled and the size selected to $320 \mathrm{bp} \pm 5 \%$ using the Pippin HT instrument.

Cluster generation and sequencing. TruSeq libraries (Illumina) were hybridized to a NextSeq (single read; Illumina). Clustering occurred on-board, where the bound library molecules were clonally amplified and sequenced using Illumina's sequencing by synthesis (SBS) chemistry. NextSeq uses 2-color chemistry to image the clusters. Upon completion of read 1, a 7-bp index read is performed in the case of single indexed libraries. If dual-indexing was used during library preparation, 2 separate 8- or 10-bp index reads were performed. Finally, clustered library fragments were resynthesized in the reverse direction, thus producing the template for a paired-end read 2.

Primary analysis and demultiplexing. Base call (.bcl) files for each cycle of sequencing were generated using Illumina Real Time Analysis (RTA) software. The base call files and run folders are streamed to servers maintained at the Minnesota Supercomputing Institute. Primary analysis and demultiplexing were performed using Illumina's bcl2fastq software, version 2.20. The end result of the bcl2fastq workflow was demultiplexed FASTQ files that were released.

RNA-Seq data analyses. 7-bp FastQ single-end reads ( $n=11.7$ million per sample) were trimmed using Trimmomatic (version 0.33) enabled with the optional "-q" option; 3-bp sliding-window trimming was from the $3^{\prime}$ end requiring minimum Q30. Quality control checks on raw sequence data for each sample were performed with Babraham Bioinformatics FastQC (version 0.11.7). Read mapping was performed with HISAT2 (version 2.1.0) using the mouse UCSC genome (mm10) as a reference. Gene quantification was done via Subread (feature Counts, version 1.4.6) for raw read counts. Differentially expressed genes were identified with the edgeR (negative binomial) feature in CLCGWB, version 10.1.2 (QIAGEN) using raw read counts. We filtered the generated list on the basis of a minimum 1.5 absolute fold change and a FDR-corrected $P$ value of less than 0.001. Complete RNA-Seq data were deposited in the NCBI's Gene Expression Omnibus (GEO) database (GSE131936; https://www.ncbi.nlm.nih.gov/geo/ query/acc.cgi .acc $=$ GSE131936).

\section{Statistics}

Data were analyzed using a 2-tailed Student's $t$ test. A $P$ value of less than 0.05 was considered significant. Statistical significance for survival studies was calculated using the log-rank (Mantel-Cox) test with a $95 \%$ CI. Results in the graphs are presented as the mean \pm SEM.

\section{Study approval}

All animal experiments were approved by the IACUC of the University of Iowa (protocol 6081822).

\section{Author contributions}

RC and SP designed the research studies. RC, ARF, JZ, and CWL conducted experiments and acquired data. RC, JEA, RS, PBM, DKM, and SP analyzed data. MM provided reagents. RC and SP wrote the manuscript.

\section{Acknowledgments}

We thank Josalyn Cho for careful review of this manuscript. We thank Michael Gale Jr. (University of Washington), West- 
ley Reeves, and Shizuo Akira for providing the $M A V S^{-/-}$and $T L R 7^{-1}$ mice. This work was supported in part by grants from the NIH (PO1AI060699 and RO1AI129269, to SP and R21AG060222, to RC).

Address correspondence to: Stanley Perlman, Department of Microbiology and Immunology, BSB 3-712, University of Iowa, Iowa City, Iowa 52242, USA. Phone: 319.335.8549; Email:
Stanley-perlman@uiowa.edu; Or to: Rudragouda Channappanavar, Departments of Acute and Tertiary Care, Microbiology and Immunology, 701E Molecular Sciences Building, 858 Madison Avenue, Memphis, Tennessee 38163, USA. Phone: 901.448.2524; Email: rchanna1@uthsc.edu.

ARF's present address is: Department of Molecular Biosciences, University of Kansas, Lawrence, Kansas, USA.
1. Bloom DE, Black S, Rappuoli R. Emerging infectious diseases: a proactive approach. Proc Natl Acad Sci U S A. 2017;114(16):4055-4059.

2. Assiri A, et al. Hospital outbreak of Middle East respiratory syndrome coronavirus. $N$ Engl J Med. 2013;369(5):407-416.

3. Peiris JS, et al. Coronavirus as a possible cause of severe acute respiratory syndrome. Lancet. 2003;361(9366):1319-1325.

4. Su S, et al. Epidemiology, genetic recombination, and pathogenesis of coronaviruses. Trends Microbiol. 2016;24(6):490-502.

5. Assiri A, et al. Epidemiological, demographic, and clinical characteristics of 47 cases of Middle East respiratory syndrome coronavirus disease from Saudi Arabia: a descriptive study. Lancet Infect Dis. 2013;13(9):752-761.

6. Middle East respiratory syndrome coronavirus (MERS-CoV). World Health Organization web site. http://www.who.int/emergencies/ mers-cov/en/. Updated September 21, 2018. Accessed June 14, 2019.

7. Menachery VD, et al. A SARS-like cluster of circulating bat coronaviruses shows potential for human emergence. Nat Med.2015;21(12):1508-1513.

8. Ge XY, et al. Isolation and characterization of a bat SARS-like coronavirus that uses the ACE2 receptor. Nature. 2013;503(7477):535-538.

9. de Wit E, et al. Middle East respiratory syndrome coronavirus (MERS-CoV) causes transient lower respiratory tract infection in rhesus macaques. Proc Natl Acad Sci U S A. 2013;110(41):16598-16603.

10. Li K, et al. Mouse-adapted MERS coronavirus causes lethal lung disease in human DPP4 knockin mice. Proc Natl Acad Sci U S A. 2017;114(15):E3119-E3128.

11. Cockrell AS, et al. A mouse model for MERS coronavirus-induced acute respiratory distress syndrome. Nat Microbiol. 2016;2:16226.

12. Baseler LJ, et al. An acute immune response to Middle East respiratory syndrome coronavirus replication contributes to viral pathogenicity. Am J Pathol. 2016;186(3):630-638.

13. van den Brand JM, Haagmans BL, van Riel D, Osterhaus AD, Kuiken T. The pathology and pathogenesis of experimental severe acute respiratory syndrome and influenza in animal models. J Comp Pathol. 2014;151(1):83-112.

14. Channappanavar R, et al. Dysregulated type I interferon and inflammatory monocytemacrophage responses cause lethal pneumonia in SARS-CoV-infected mice. Cell Host Microbe. 2016;19(2):181-193.

15. Schneider WM, Chevillotte MD, Rice CM. Interferon-stimulated genes: a complex web of host defenses. Annu Rev Immunol. 2014;32:513-545.

16. Totura AL, Baric RS. SARS coronavirus pathogenesis: host innate immune responses and viral antagonism of interferon. Curr Opin Virol. 2012;2(3):264-275.

17. Omrani AS, et al. Ribavirin and interferon alfa-2a for severe Middle East respiratory syndrome coronavirus infection: a retrospective cohort study. Lancet Infect Dis. 2014;14(11):1090-1095.

18. Kim UJ, Won EJ, Kee SJ, Jung SI, Jang HC. Combination therapy with lopinavir/ritonavir, ribavirin and interferon- $\alpha$ for Middle East respiratory syndrome. Antivir Ther (Lond). 2016;21(5):455-459.

19. Loutfy MR, et al. Interferon alfacon-1 plus corticosteroids in severe acute respiratory syndrome: a preliminary study. JAMA 2003;290(24):3222-3228.

20. Gao L, et al. A randomized controlled trial of low-dose recombinant human interferons alpha-2b nasal spray to prevent acute viral respiratory infections in military recruits. Vaccine. 2010;28(28):4445-4451.

21. Davidson S, Crotta S, McCabe TM, Wack A. Pathogenic potential of interferon $\alpha \beta$ in acute influenza infection. Nat Commun. 2014;5:3864.

22. Davidson S, Maini MK, Wack A. Diseasepromoting effects of type I interferons in viral, bacterial, and coinfections. J Interferon Cytokine Res. 2015;35(4):252-264.

23. Wilson EB, et al. Blockade of chronic type I interferon signaling to control persistent LCMV infection. Science. 2013;340(6129):202-207.

24. Teijaro JR, et al. Persistent LCMV infection is controlled by blockade of type I interferon signaling. Science. 2013;340(6129):207-211.

25. Stockman LJ, Bellamy R, Garner P. SARS: systematic review of treatment effects. PLoS Med. 2006;3(9):e343.

26. Shalhoub S, et al. IFN- $\alpha 2$ a or IFN- $\beta 1$ a in combination with ribavirin to treat Middle East respiratory syndrome coronavirus pneumonia: a retrospective study. J Antimicrob Chemother. 2015;70(7):2129-2132.

27. Al-Tawfiq JA, Momattin H, Dib J, Memish ZA. Ribavirin and interferon therapy in patients infected with the Middle East respiratory syndrome coronavirus: an observational study. Int J Infect Dis. 2014;20:42-46.

28. Cervantes-Barragan L, et al. Control of coronavirus infection through plasmacytoid dendritic-cell-derived type I interferon. Blood. 2007;109(3):1131-1137.

29. Roth-Cross JK, Bender SJ, Weiss SR. Murine coronavirus mouse hepatitis virus is recognized by MDA 5 and induces type I interferon in brain macrophages/microglia.J Virol.
2008;82(20):9829-9838

30. Zarember KA, Godowski PJ. Tissue expression of human Toll-like receptors and differential regulation of Toll-like receptor mRNAs in leukocytes in response to microbes, their products, and cytokines. JImmunol. 2002;168(2):554-561.

31. Ioannidis I, Ye F, McNally B, Willette M, Flaño E. Toll-like receptor expression and induction of type I and type III interferons in primary airway epithelial cells. J Virol. 2013;87(6):3261-3270.

32. Zielecki F, et al. Human cell tropism and innate immune system interactions of human respiratory coronavirus EMC compared with those of severe acute respiratory syndrome coronavirus. JVirol. 2013;87(9):5300-5304.

33. Peiris JS. Severe acute respiratory syndrome (SARS). J Clin Virol. 2003;28(3):245-247.

34. Franks TJ, et al. Lung pathology of severe acute respiratory syndrome (SARS): a study of 8 autopsy cases from Singapore. Hum Pathol. 2003;34(8):743-748.

35. Ng DL, et al. Clinicopathologic, immunohistochemical, and ultrastructural findings of a fatal case of Middle East respiratory syndrome coronavirus infection in the United Arab Emirates, April 2014. Am J Pathol. 2016;186(3):652-658.

36. Zhao J, et al. Rapid generation of a mouse model for Middle East respiratory syndrome. Proc Natl Acad Sci U S A. 2014;111(13):4970-4975.

37. Kaner RJ, et al. Modification of the genetic program of human alveolar macrophages by adenovirus vectors in vitro is feasible but inefficient, limited in part by the low level of expression of the coxsackie/adenovirus receptor. Am J Respir Cell Mol Biol. 1999;20(3):361-370.

38. Law HK, et al. Chemokine up-regulation in SARS-coronavirus-infected, monocytederived human dendritic cells. Blood. 2005;106(7):2366-2374.

39. Cheung CY, et al. Cytokine responses in severe acute respiratory syndrome coronavirus-infected macrophages in vitro: possible relevance to pathogenesis. J Virol. 2005;79(12):7819-7826.

40. Gu J, et al. Multiple organ infection and the pathogenesis of SARS. J Exp Med. 2005;202(3):415-424.

41. Nicholls JM, et al. Lung pathology of fatal severe acute respiratory syndrome. Lancet. 2003;361(9371):1773-1778.

42. Cervantes-Barragán L, et al. Type I IFN-mediated protection of macrophages and dendritic cells secures control of murine coronavirus infection. Jimmunol. 2009;182(2):1099-1106.

43. Koestner W, et al. Interferon-beta expression and type I interferon receptor signaling of hepatocytes prevent hepatic necrosis and virus dissemination in Coxsackievirus B3-infected mice. PLoS 
Pathog. 2018;14(8):e1007235.

44. Monto AS, Albrecht JK, Schwartz SA. Demonstration of dose-response relationship in seasonal prophylaxis of respiratory infections with alpha-2b interferon. Antimicrob Agents Chemother. 1988;32(1):47-50.

45. Farr BM, Gwaltney JM, Adams KF, Hayden FG. Intranasal interferon-alpha 2 for prevention of natural rhinovirus colds. Antimicrob Agents Chemother. 1984;26(1):31-34.

46. Kim ES, et al. Clinical progression and cytokine profiles of Middle East respiratory syndrome coronavirus infection. J Korean Med Sci. 2016;31(11):1717-1725.

47. Min CK, et al. Comparative and kinetic analysis of viral shedding and immunological responses in MERS patients representing a broad spectrum of disease severity. Sci Rep. 2016;6:25359.

48. Kolumam GA, Thomas S, Thompson LJ, Sprent J, Murali-Krishna K. Type I interferons act directly on CD8 T cells to allow clonal expansion and memory formation in response to viral infection. JExp Med. 2005;202(5):637-650.

49. Curtsinger JM, Valenzuela JO, Agarwal P, Lins D, Mescher MF. Type I IFNs provide a third signal to CD8 $\mathrm{T}$ cells to stimulate clonal expansion and differentiation. JImmunol. 2005;174(8):4465-4469.

50. Zhao J, Zhao J, Legge K, Perlman S. Age-related increases in $\mathrm{PGD}(2)$ expression impair respiratory DC migration, resulting in diminished $\mathrm{T}$ cell responses upon respiratory virus infection in mice. JClin Invest. 2011;121(12):4921-4930.

51. Zhao J, Zhao J, Perlman S. T cell responses are required for protection from clinical disease and for virus clearance in severe acute respiratory syndrome coronavirus-infected mice. J Virol. 2010;84(18):9318-9325.

52. Coleman $\mathrm{CM}$, et al. $\mathrm{CD}^{+} \mathrm{T}$ cells and macrophages regulate pathogenesis in a mouse model of Middle East respiratory syndrome. J Virol. 2017;91(1):e01825-16.
53. Seo SU, et al. Type I interferon signaling regulates Ly6C(hi) monocytes and neutrophils during acute viral pneumonia in mice. PLoS Pathog. 2011;7(2):e1001304.

54. Tynell J, et al. Middle East respiratory syndrome coronavirus shows poor replication but significant induction of antiviral responses in human monocyte-derived macrophages and dendritic cells. J Gen Virol. 2016;97(2):344-355.

55. Zhou J, et al. Active replication of Middle East respiratory syndrome coronavirus and aberrant induction of inflammatory cytokines and chemokines in human macrophages: implications for pathogenesis. J Infect Dis. 2014;209(9):1331-1342.

56. Chu H, et al. Middle East respiratory syndrome coronavirus efficiently infects human primary T lymphocytes and activates the extrinsic and intrinsic apoptosis pathways. J Infect Dis. 2016;213(6):904-914.

57. Scheuplein VA, et al. High secretion of interferons by human plasmacytoid dendritic cells upon recognition of Middle East respiratory syndrome coronavirus. JVirol. 2015;89(7):3859-3869.

58. Bang FB, Warwick A. Mouse macrophages as host cells for the mouse hepatitis virus and the genetics basis of their susceptibility. Proc Natl Acad Sci U S A. 1960;46(8):1065-1075.

59. Kantoch M, Warwick A, Bang FB. The cellular nature of genetic susceptibility to a virus. JExp Med.1963;117:781-798.

60. Koyama S, et al. Differential role of TLR- and RLR-signaling in the immune responses to influenza A virus infection and vaccination. JImmunol. 2007;179(7):4711-4720.

61. Goritzka M, et al. Alveolar macrophage-derived type I interferons orchestrate innate immunity to RSV through recruitment of antiviral monocytes. JExp Med. 2015;212(5):699-714.

62. Siu KL, et al. Middle east respiratory syndrome coronavirus 4 a protein is a double-stranded RNA-binding protein that suppresses
PACT-induced activation of RIG-I and MDA5 in the innate antiviral response. J Virol. 2014;88(9):4866-4876.

63. Gralinski LE, et al. Complement activation contributes to severe acute respiratory syndrome coronavirus pathogenesis. MBio. 2018;9(5):e01753-18.

64. Wang Y, et al. Timing and magnitude of type I interferon responses by distinct sensors impact CD8 $\mathrm{T}$ cell exhaustion and chronic viral infection. Cell Host Microbe. 2012;11(6):631-642.

65. Davidson S, et al. IFN $\lambda$ is a potent anti-influenza therapeutic without the inflammatory side effects of IFN $\alpha$ treatment. EMBO Mol Med. 2016;8(9):1099-1112.

66. Falzarano D, et al. Treatment with interferon$\alpha 2 \mathrm{~b}$ and ribavirin improves outcome in MERS-CoV-infected rhesus macaques. Nat Med. 2013;19(10):1313-1317.

67. Khalid M, Al Rabiah F, Khan B, Al Mobeireek A, Butt TS, Al Mutairy E. Ribavirin and interferon$\alpha 2 \mathrm{~b}$ as primary and preventive treatment for Middle East respiratory syndrome coronavirus: a preliminary report of two cases. Antivir Ther (Lond). 2015;20(1):87-91.

68. Meyerholz DK, Beck AP. Principles and approaches for reproducible scoring of tissue stains in research. Lab Invest. 2018;98(7):844-855.

69. Mack M, et al. Expression and characterization of the chemokine receptors CCR2 and CCR 5 in mice. JImmunol. 2001;166(7):4697-4704.

70. Zhou H, Zhao J, Perlman S. Autocrine interferon priming in macrophages but not dendritic cells results in enhanced cytokine and chemokine production after coronavirus infection. MBio. 2010;1(4):e00219-10.

71. Lee PY, et al. Type I interferon modulates monocyte recruitment and maturation in chronic inflammation. Am J Pathol. 2009;175(5):2023-2033.

72. Zhao J, et al. Intranasal treatment with poly $(\mathrm{I} \cdot \mathrm{C})$ protects aged mice from lethal respiratory virus infections. JVirol. 2012;86(21):11416-11424. 